\title{
Adaptive Fuzzy Sliding Mode Control for a 3-DOF Parallel Manipulator with Parameters Uncertainties
}

\author{
Haiqiang Zhang $\mathbb{D}^{1,2}$ Hairong Fang $\mathbb{D}^{1},{ }^{1}$ Dan Zhang, ${ }^{2}$ Xueling Luo, ${ }^{2}$ and Qi Zou ${ }^{2}$ \\ ${ }^{1}$ School of Mechanical, Electronic and Control Engineering, Beijing Jiaotong University, Beijing, China \\ ${ }^{2}$ Lassonde School of Engineering, York University, Ontario, M3J 1P3, Canada
}

Correspondence should be addressed to Haiqiang Zhang; haiqiang@yorku.ca and Hairong Fang; hrfang@bjtu.edu.cn

Received 5 June 2019; Revised 26 July 2019; Accepted 13 August 2019; Published 23 January 2020

Academic Editor: Yongping Pan

Copyright ( 92020 Haiqiang Zhang et al. This is an open access article distributed under the Creative Commons Attribution License, which permits unrestricted use, distribution, and reproduction in any medium, provided the original work is properly cited.

\begin{abstract}
Parallel manipulators possess the advantages of being compact structure, high stiffness, stability and high accuracy, so such parallel manipulators have been widely employed in application fields as diverse as parallel kinematic machine, motion simulator platform, medical rehabilitation device, and so on. Due to the complexity of the closed-loop structural system, an accurate dynamic model is very difficult to be derived in the absence of some uncertainties parameters and external disturbances. In order to improve the trajectory tracking accuracy with time-varying and nonlinear parameters, this paper addresses the design and implement of adaptive fuzzy sliding mode control (AFSMC) for a three-degree-of-freedom (DOF) parallel manipulator, where internal force term can be linearly separated into a regression matrix and a parameter vector that contains the estimated errors. Furthermore, fuzzy inference unit is utilized to modify the gain parameters in real-time by using the state feedback from the task space and the adaptive law is performed to update uncertainties in dynamic parameters. The proposed controller is deduced in the sense of Lyapunov theory to guarantee the stability while improving the trajectory tracking performance. Finally, simulation experiment results demonstrate that the proposed control method is insensitive to uncertainties and disturbances and permits to decrease the requirement for the bound of these uncertainties, which validate the effectiveness of the developed control method and exhibit good trajectory tracking performance compared with sliding mode control (SMC) and fuzzy sliding model control (FSMC).
\end{abstract}

\section{Introduction}

Parallel manipulators are widely believed to be an interdisciplinary research area which becomes an outstanding field attracting more and more attention from both industrial and academic domains. Some researchers began to focus on parallel manipulators because of the advantages they hold over their serial counterparts: high stiffness, high accuracy, quick response speed, and high payload to weight ratio. A large number of new architectures dealing with the kinematics and dynamics of parallel manipulators have been proposed in academic $[1,2]$, while some of them have been implemented to practical applications, e.g., parallel kinematic machine [3], motion simulator platform [4], surgical medical equipment [5], dexterous hands [6], etc. However, in order to achieve potential advantages over serial manipulators, parallel manipulators still require improvement in the design, modeling, analysis, and optimization, while the development of control schemes also provides a field for improving the performance of the parallel manipulators to some extent [7].

Due to the complexity of the closed-loop structural system, the dynamic model of parallel manipulator is more complicated than it is in serial manipulator. In [8], the simplified model was obtained by neglecting the friction and their deformation, and based on an assumption that the rigidity of the moving platform is much greater than other components. However, the assumption cannot be generalized for all the parallel manipulators. Liang et al. [9] developed a nonlinear dynamic modeling of a redundantly actuated parallel manipulator based on the flexible multi-body dynamics theory. It is mentioned that the closed-loop dynamic control of the parallel mechanisms is a challenging domain due to the high complexity of their dynamic behavior [10-12].

In industrial applications, the parallel manipulators need to achieve trajectory tracking for control implementation, one of the most common control techniques applied to the 
parallel manipulators is the family of PID controllers (including PI and PD controllers) which are mainly designed in terms of kinematics [13-15]. In order to improve the control precision of the existing PID controller, a variable PID parameter controller optimized by a genetic algorithm was proposed by Sheng in [16]. Zhan and Kao [17] proposed a hybrid damped resolved-acceleration control (HDRAC) scheme, which considered both damped acceleration and velocity as a way to remove these unnecessary velocities, a modified particle swarm optimization (MPSO) was adopted to obtain simple and effective estimated damping value, and the illustrative examples of the 3RPS parallel manipulator are provided to verify the utility of the proposed control scheme. But as the parallel manipulator is multi-variable and multi-parameter coupling nonlinear system, its dynamic model is very complicated, and external disturbances such as friction, environment noise are always inevitable during the motion process. Therefore, the dynamic model has some uncertainties and the traditional dynamic control generally cannot effectively solve the uncertainty problem. In this case, the advanced controllers can improve the trajectory tracking performance, high kinematic accuracy, and system stability [18]. Sliding mode control (SMC) is a robust method that has an appropriate performance to overcome the disturbance and uncertainties, which is extremely effective when the bounds of disturbances and uncertainties are known [19]. A sliding mode controller is designed using a presumed upper bound of uncertainties as a control parameter, which may generate large amplitude chattering in the control input. If the uncertainty is in the allowable range of the designed controller, the controller will perform well. But if the uncertainty exceeds the limits, the system will become unstable or the controller performance will be degraded greatly. To overcome the aforementioned problems in the design of SMC, fuzzy sliding mode control is proposed in [20] as a model-free controller scheme, which is an alternative effective approach to control such system for its advantage of independent on a precise mathematical model, as well as good robustness and nonlinear characteristics. A modified robust dynamic control (MRDC) method was proposed for a 2-DOF planar parallel robot to realize high precision and high speed cooperation motion. The designed control law was insensitive to the noise signal, which can help to improve error convergence ratio without causing undesirable chattering in [21]. Cazalilla et al. [22] developed an adaptive control of a 3-DOF parallel manipulator by considering the rigid parameters, the friction parameters, the actuator dynamics, and a combination of the former parameters. An adaptive controller was proposed on a planar parallel robot with uncertainties in dynamic and kinematic parameters and the simulation illustrated that the adaptation of the kinematic and dynamic parameters can improve the performance by adjusting the direction of resultant internal force [23, 24]. Le et al. [25] presented a novel chattering free neuro-sliding mode controller for two degrees of planar parallel manipulator which had a complicated dynamic model, including frictional uncertainties, modeling uncertainties, and external disturbances. The simulation results showed the effectiveness for the tracking control of the 2-DOF parallel manipulator, and the control system can hold small errors and good robustness against uncertainties and external disturbances.

In addition, the combinations of fuzzy sliding mode controller and adaptive controller have also attracted great interest in the academic fields. Qi et al. [26] proposed a fuzzy adaptive supervisory controller (FASC) for the 4-DOF parallel manipulator to reduce the chattering, and the comparative analysis with the sliding surface control (SMC) indicated that the improved control can reduce greatly the chattering phenomenon and possess good robustness against the parameters uncertainties and external disturbances. Filabi et al. [27] proposed a fuzzy adaptive sliding mode controller for trajectory tracking with considering dynamics of electromechanical actuators, and several simulation cases demonstrated that the proposed control strategy can achieve favorable control performance with regard to uncertainties, nonlinearities, and external disturbances. An adaptive robust controller integrated with online dead-zone estimation was proposed in [28] and the nonlinear characteristics and unknown parameters in the dynamic model can be estimated and compensated by online estimation methods, and the unknown parameters converged to the actual values within finite time. A new extended adaptive fuzzy sliding mode controller and robust observer method was applied for position control of a Stewart manipulator whose parameters are strongly state-dependent and complex, so as to navigate toward the desired trajectory in the presence of uncertainty and unknown upper bound, the upper bound of the uncertainty required to be progressively estimated utilizing the designed adaptation rules [29]. A large number of literatures are presented, and the researches on the control of the parallel manipulators are most concentrated on the planar parallel manipulator and six degrees of freedom parallel manipulator. However, the control schemes on the spatial redundantly actuated parallel manipulator are still merely, furthermore, the conventional PID control is unavailable to be implemented on the designed parallel manipulator, so the advanced control algorithm is carried out on the redundantly actuated parallel manipulator to perform trajectory tracking in this paper.

The main contribution of this paper is to propose an alternative adaptive fuzzy sliding mode control algorithm for a 3-DOF redundantly actuated parallel manipulator. The control algorithm combines the advantages of conventional sliding mode controller and fuzzy logic controller. The stability of the control system is theoretically proven in terms of Lyapunov method, and the tracking errors can converge to zero asymptotically. The parameters uncertainties of the control system are compensated by the adaptive control law and the unknown disturbances are rejected by the fuzzy self-tuning robust control law. The simulation experiment results demonstrate that the developed controller can exhibit excellent tracking performance compared with the two other controllers. Furthermore, the appearing characteristics of the proposed controller are fast convergence, high precision, and high robustness as compared with aforementioned controllers.

The paper is organized as follows. In Section 2, a 3-DOF parallel manipulator is proposed as the research object. The kinematic and dynamic analysis is carried out, the mapping relation related to the velocity between actuator and endeffector is obtained, and the dynamics equation is established 


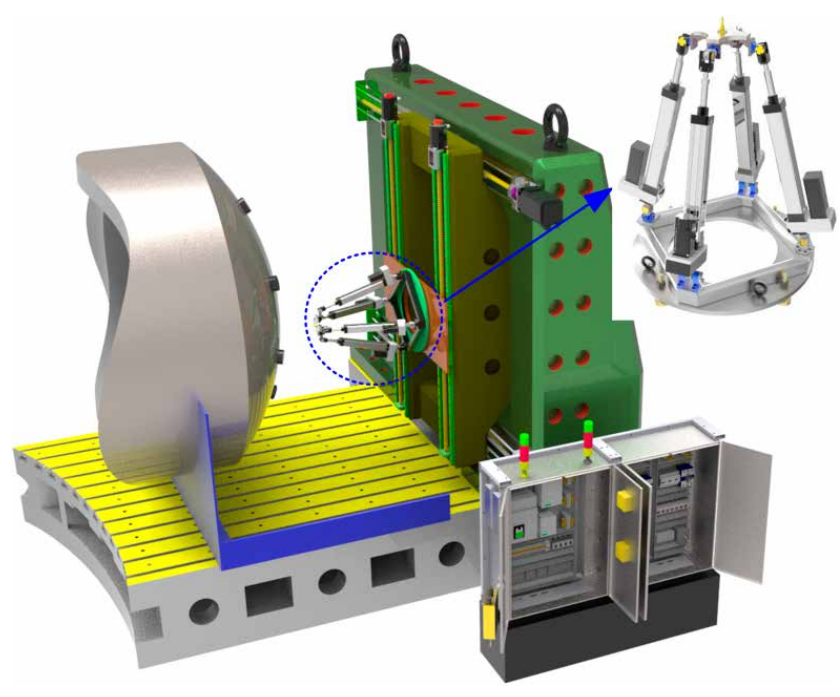

FIGURE 1: A serial-parallel hybrid kinematic machine.

as well. In Section 3, the designed adaptive fuzzy sliding mode controller is introduced and derived in detail based on the sliding mode controller and fuzzy sliding mode controller. The simulation experiment results demonstrate the proposed adaptive fuzzy sliding mode controller can achieve the best trajectory tracking performance compared with other two controllers in Section 4. Finally in Section 5, the several remarks and further work are addressed.

\section{Kinematic and Dynamic Model of the Parallel Manipulator}

In this paper, a serial-parallel hybrid kinematic machine tool has been presented, which is a combination of 3-DOF parallel manipulator and two long $X-Y$ tracks. And it can be applied to high speed machining for a large heterogeneous complex freedom surface in the aerospace field, as shown in Figure 1. The main unit module is a 2RPU-2SPR parallel manipulator, and its architectural diagram is depicted in Figure 2, which consists of an upper platform, a fixed base platform, and four actuated limbs connecting the base and upper platform. The parallel manipulator can achieve three degrees of freedom on the basis of the simultaneous movement of the four prismatic actuators. It is a remarkable fact that the proposed parallel manipulator belongs to parallel manipulator with redundant actuation due to the number of actuated joints is greater than that of the degree of freedom.

The geometric model of the parallel manipulator and its vector diagram can be seen in Figure 2, where the radius of the upper platform is defined as $r a$, likewise, the parameter of the fixed base platform is defined as $r b$, respectively. For purpose of analysis, Figure 2 shows the coordinates of the parallel manipulator; positions of the upper platform and base can be uniquely defined by the coordinates of the four kinematic joints. The vector $a_{\mathrm{i}}$ describes the positions of four vertices with respect to the coordinate system $A-u v w$ which is attached on the upper platform. Vector $b_{\mathrm{i}}$ describes the positions of four vertices with respect to the coordinate system $B-x y z$, which is attached on

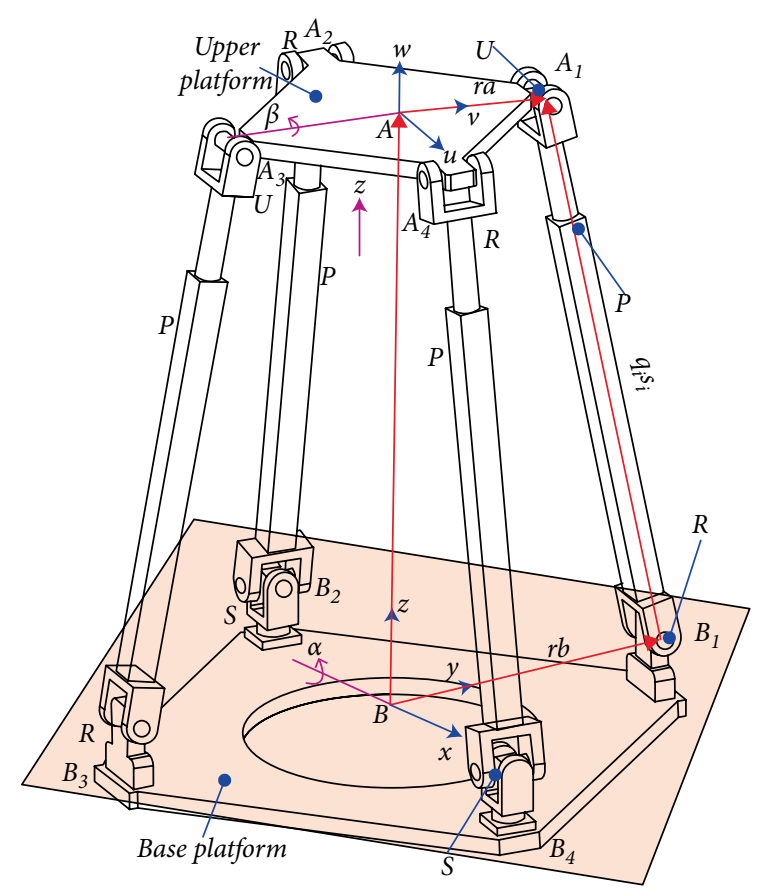

Figure 2: Schematic diagram of 3-DOF parallel manipulator.

the base platform. Let the parameters $(z, \alpha, \beta)$ be as the three independent variables to describe the translation and rotations of the upper platform, i.e., the three independent degrees of freedom. The mobility analysis of the parallel manipulator including initial configuration and general configuration has been addressed in detail in our previous work [30] by resorting to the screw theory and modified Grubler-Kutzbach $(G-K)$ criterion. To avoid repetition, herein, the mobility regarding the parallel manipulator is not described in this paper.

The kinematics problems of parallel manipulator can be divided into the inverse kinematics and the forward kinematics, where the inverse one means to compute the distance of prismatic actuators when the position and orientations are given, while the forward one, vice versa. In general, if given the desired trajectory of the upper platform, the inverse kinematics could be employed to transform the trajectory into the displacement of each actuator, then to formulate the dynamic equation in the joint space. The other way is that the measured displacement of each prismatic joint can be transformed into task space by utilizing the forward kinematics, and then the dynamic equation can be developed in the task operational space. However, there is another simpler approach that the dynamic equation can be developed in task space by means of the camera technique avoiding the complicated forward kinematic solutions $[31,32]$.

The position and orientation of the upper platform with respect to the coordinate frame $B-x y z$ can be described by a matrix $\mathbf{T}$ that contains the rotational transformation matrix $\mathbf{R}$ and the translation vector $\mathbf{p}$.

$$
\mathbf{T}=\left[\begin{array}{ll}
\mathbf{R} & \mathbf{p} \\
0 & 1
\end{array}\right]=\left[\begin{array}{ccc|c}
c \beta & 0 & s \beta & 0 \\
s \alpha s \beta & c \alpha & -s \alpha c \beta & -h s \alpha \\
-s \beta c \alpha & s \alpha & c \alpha c \beta & h c \alpha \\
\hline 0 & 0 & 0 & 1
\end{array}\right],
$$


where $s$ and $c$ are the abbreviation of sine and cosine, respectively, and $h$ represents the motion distance in the direction of perpendicular to the upper platform.

The translation vector $\mathbf{p}$ can be also written as

$$
\begin{aligned}
& x=0, \\
& y=-h s \alpha, \\
& z=h s \alpha .
\end{aligned}
$$

So the coupling relationship in Equation (3) can be deduced from (2)

$$
y=-z \tan \alpha .
$$

The length $q_{i}$ of the $i$-th actuated limb can be derived as

$$
q_{i}=\left\|\frac{\mathbf{p}+\mathbf{a}_{i}-\mathbf{b}_{i}}{\mathbf{s}_{i}}\right\|_{2},
$$

where $\|\cdot\|_{2}$ corresponds to the standard Euclid norm, and $s_{i}$ is the unit vector along the $i$-th prismatic linear actuator.

The derivative of Equation (4) with respect to time can be written in a matrix form as

$$
\dot{\mathbf{q}}=\mathbf{J} \dot{\mathbf{X}}
$$

where the matrix $\mathbf{J} \in R^{4 \times 3}$ is the Jacobian matrix that indicates the velocity mapping relationship between actuated joints and the end-effector.

The dynamic model of the parallel manipulator can be regarded as the motion equation of the closed-loop system. It represents the relationship between the driving force/torque and the variables of joints. The dynamic equations can be established by means of the principle of virtual work [33], the Lagrange method [34], the Newton-Euler method [35] and Kane formulation [36], and so on. Assuming the upper platform is a rigid body, and ignoring friction force of the kinematic joints as well as the uncertainties and external disturbances. The dynamic model can be established in terms of the principle of virtual work as follows [37]

$$
\begin{gathered}
\mathbf{F}_{X}=\mathbf{M}(\mathbf{X}) \ddot{\mathbf{X}}+\mathbf{C}(\mathbf{X}, \dot{\mathbf{X}}) \dot{\mathbf{X}}+\mathbf{G}(\mathbf{X}), \\
\tau_{a}=\mathbf{M}(\mathbf{q}) \ddot{\mathbf{q}}+\mathbf{C}(\mathbf{q}) \dot{\mathbf{q}}+\mathbf{G}(\mathbf{q}),
\end{gathered}
$$

where $\mathbf{X}=\left[\begin{array}{lll}z & \alpha & \beta\end{array}\right]^{T}$ represents the independent Cartesian task space vector, $\mathbf{q}=\left[\begin{array}{llll}q_{1} & q_{2} & q_{3} & q_{4}\end{array}\right]^{T}$ is the joint space vector, $F_{x}$ is the force vector in the task space, $\boldsymbol{\tau}_{a}$ is the force vector in joint space, $\mathbf{M}(\mathbf{X})$ and $\mathbf{M}(\mathbf{q})$ are the inertia matrix, $\mathbf{C}(\mathbf{X})$ and $\mathbf{C}(\mathbf{q})$ are the Coriolics and Centrifugal coefficient matrix, and $\mathbf{G}(\mathbf{X})$ and $\mathbf{G}(\mathbf{q})$ are the gravity vector.

The dual relationship between the force in task space and in the joint space can be denoted by resorting to the Jacobain matrix $\mathbf{J}$ of the kinematics.

$$
\mathbf{F}_{X}=\mathbf{J}^{T} \boldsymbol{\tau}_{a}
$$

Therefore, the driving force $\tau_{a}$ can be expressed as

$$
\boldsymbol{\tau}_{a}=\left[\mathbf{J}^{T}\right]^{+}[\mathbf{M}(\mathbf{X}) \ddot{\mathbf{X}}+\mathbf{C}(\mathbf{X}, \dot{\mathbf{X}}) \dot{\mathbf{X}}+\mathbf{G}(\mathbf{X})]
$$

where $[\cdot]^{+}$denotes the Moore-Penrose inverse matrix. The Moore-Penrose inverse of the matrix $\mathbf{J}^{T}$ can be denoted as
$\left(\mathbf{J}^{T}\right)^{+}=\mathbf{J}\left(\mathbf{J}^{T} \mathbf{J}\right)^{-1}$ and the condition $\left(\mathbf{J}^{T}\right)^{+} \mathbf{J}^{T}=\mathbf{E}$ should be satisfied.

In general, due to the presence of structured and unstructured uncertainties and the external disturbance, such as mass, moment of inertia, and friction, it is very difficult to establish an accurate dynamic model, but if the modeling errors caused by the uncertainties are bounded with known functions, then we can utilize modeling errors between the measured value and the real values. Therefore, the dynamic equation can be derived by combining the estimated values with parameters errors.

$$
\mathbf{F}_{X}=\mathbf{J}^{T} \tau_{a}=\hat{\mathbf{M}}(\mathbf{X}) \ddot{\mathbf{X}}+\hat{\mathbf{C}}(\mathbf{X}, \dot{\mathbf{X}}) \dot{\mathbf{X}}+\hat{\mathbf{G}}(\mathbf{X}),
$$

where $\hat{\mathbf{M}}(\mathbf{X}), \hat{\mathbf{C}}(\mathbf{X}, \dot{\mathbf{X}})$ and $\hat{\mathbf{G}}(\mathbf{X})$ are the estimated parameter value of $\mathbf{M}(\mathbf{X}), \mathbf{C}(\mathbf{X}, \dot{\mathbf{X}})$, and $\mathbf{G}(\mathbf{X})$, respectively.

Equation (9) subtracting Equation (10), yields

$$
\mathbf{F}_{X}=\mathbf{J}^{T} \boldsymbol{\tau}_{a}=\mathbf{M}(\mathbf{X}) \ddot{\mathbf{X}}+\mathbf{C}(\mathbf{X}, \dot{\mathbf{X}}) \dot{\mathbf{X}}+\mathbf{G}(\mathbf{X})-\boldsymbol{\tau}_{d}
$$

In which

$$
\boldsymbol{\tau}_{d}=(\mathbf{M}-\hat{\mathbf{M}}) \ddot{\mathbf{X}}+(\mathbf{C}-\hat{\mathbf{C}}) \dot{\mathbf{X}}+(\mathbf{G}-\hat{\mathbf{G}})+\boldsymbol{\tau}_{d}^{\prime} .
$$

Here $\tau_{d}$ is the uncertainty term caused by the parameters errors and external disturbance. as $\mathbf{G}$.

For brevity, we denote $\mathbf{M}(\mathbf{X})$ as $\mathbf{M}, \mathbf{C}(\mathbf{X}, \dot{\mathbf{X}})$ as $\mathbf{C}$, and $\mathbf{G}(\mathbf{X})$

Simultaneously, the parameters errors can be defined as

$$
\tilde{\mathbf{M}}=\mathbf{M}-\hat{\mathbf{M}}, \tilde{\mathbf{C}}=\mathbf{C}-\hat{\mathbf{C}}, \tilde{\mathbf{G}}=\mathbf{G}-\hat{\mathbf{G}} \text {. }
$$

\section{Controller Design}

The purpose of the control is that the parallel manipulator is at the defined control law $\mathbf{u}$, and the upper platform can still move according to the predicted trajectory and maintain the stability of the system when there are some uncertainties such as modeling errors, parameters errors, external disturbances, unknown loads, etc.

We proposed an adaptive fuzzy sliding mode controller (AFSMC), in order to validate the effectiveness, and comparisons with other control schemes including Sliding Mode Control (SMC) and Fuzzy Sliding Mode Control (FSMC) were conducted. In the following, three controllers: SMC, FSMC, and AFSMC, will be designed to implement the desired trajectory tracking.

3.1. Sliding Mode Control. Define $\mathbf{X}_{d}, \dot{\mathbf{X}}_{d}$ is the desired state and velocity, $\mathbf{X}$ and $\dot{\mathbf{X}}$ is the actual state and velocity, and $\mathbf{e}$ and $\dot{\mathbf{e}}$ are the tracking error and the derivate of the tracking error, respectively.

$$
\begin{aligned}
& \mathbf{e}=\mathbf{X}_{d}-\mathbf{X}, \\
& \dot{\mathbf{e}}=\dot{\mathbf{X}}_{d}-\dot{\mathbf{X}} .
\end{aligned}
$$

Define the sliding surface function $\mathbf{s}$ as

$$
\mathbf{s}=\dot{\mathbf{e}}+\lambda \mathbf{e},
$$

where $\lambda \in R^{3 \times 3}$ is the positive matrix. 
From Equation (15), we can see that if the designed control law is reasonable, and the sliding surface function $\mathbf{s}$ will be close to zero asymptotically, then the error $\mathbf{e} \rightarrow 0$, and $\dot{\mathbf{e}} \rightarrow 0$, and the closed-loop system can be globally stable.

Differentiating both sides of Equation (15), yields

$$
\dot{\mathbf{s}}=\ddot{\mathbf{e}}+\lambda \dot{\mathbf{e}} .
$$

Define the Lyapunov function candidate as follows

$$
V=\frac{1}{2} \mathbf{s}^{T} \mathbf{M s} .
$$

Due to $\mathbf{M}$ is symmetric and positive definite matrix.

Hence,

$$
V \geq 0 \text {. }
$$

If and only if $\mathbf{s}=\mathbf{0}$, then $V(\mathbf{0})=0$.

Note that, with another particular property of the parallel manipulator that the matrix $\dot{\mathbf{M}}-2 \mathbf{C}$ is indeed skew-symmetric matrix [38], and substituting into Equation (19), differentiating of Lyapunov function $V$ can be derived as,

$$
\begin{aligned}
\dot{V} & =s^{T}\left[\mathbf{M} \dot{\mathbf{s}}+\frac{1}{2} \dot{\mathbf{M}} \mathbf{s}\right] \\
& =\mathbf{s}^{T}[\mathbf{M}(\ddot{\mathbf{e}}+\lambda \dot{\mathbf{e}})+C(\dot{\mathbf{e}}+\lambda \mathbf{e})] \\
& =\mathbf{s}^{T}\left[\mathbf{M}\left(\ddot{X}_{d}-\ddot{X}+\lambda \dot{\mathbf{e}}\right)+C\left(\dot{X}_{d}-\dot{X}++\lambda \mathbf{e}\right)\right] \\
& =\mathbf{s}^{T}\left[\mathbf{M}\left(\ddot{X}_{d}+\lambda \dot{\mathbf{e}}\right)+C\left(\dot{X}_{d}+\lambda \mathbf{e}\right)-\mathbf{M} \ddot{X}-\mathbf{C} \dot{X}\right] \\
& =\mathbf{s}^{T}\left[\mathbf{M}\left(\ddot{X}_{d}+\lambda \dot{\mathbf{e}}\right)+C\left(\dot{X}_{d}+\lambda \mathbf{e}\right)+G(X)-\tau_{d}-u_{1}\right] .
\end{aligned}
$$

Define sliding mode control law $\mathbf{u}_{1}$

$$
\mathbf{u}_{1}=\mathbf{M}\left(\ddot{\mathbf{X}}_{d}+\lambda \dot{\mathbf{e}}\right)+\mathbf{C}\left(\dot{\mathbf{X}}_{d}+\lambda \mathbf{e}\right)+\mathbf{G}\left(\mathbf{X}_{d}\right)+\mathbf{K s}+\Lambda \operatorname{sgn}(\mathbf{s}),
$$

where $\mathbf{K}$ is positive define feedback gain matrix, and $\boldsymbol{\Lambda}$ is a constant matrix.

Substituting Equation (20) into (19), yields

$$
\dot{V}=\mathbf{s}^{T}\left[-\boldsymbol{\tau}_{d}-\Lambda \operatorname{sgn}(\mathbf{s})-\mathbf{K s}\right] \leq \mathbf{s}^{T}\left[\left|\tau_{d}\right|-\Lambda|\mathbf{s}|\right)-\mathbf{s}^{T} \mathbf{K} \mathbf{s} .
$$

If the condition $\left|\tau_{d}\right| \leq|\Lambda|$ is satisfied, then Equation (21) can be simplified to

$$
\dot{V} \leq-\mathbf{s}^{T} \mathbf{K} \mathbf{s} \leq 0 .
$$

Therefore, according to the Lyapunov stability theory, the desired trajectory of the closed-loop system will be eventually convergence to the sliding surface

$$
\mathbf{s}=\dot{\mathbf{e}}+\lambda \mathbf{e}=0 .
$$

And therefore, the error e will evolve toward zero, then the derivative of the error $\dot{e}$ will evolve zero as well if time is infinity, which means that the designed controller can guarantee the actual trajectory $\boldsymbol{X}$ to track the given $\boldsymbol{X}_{d}$, and the corresponding closed-loop system can be globally stable.

Since the range of the sgn function in (20) varies from -1 to 1 , then chattering phenomenon is inevitable around the sliding mode surface. Therefore, to further avoid the undesirable phenomenon of oscillations with finite frequency and amplitude, the discontinuous sgn function needs to be replaced by a continuous approximation saturation function in Equation (20), i.e.,

$$
\operatorname{sgn}(\mathbf{s})=\operatorname{sat}\left(\frac{\mathbf{s}}{\boldsymbol{\varepsilon}}\right)=\left\{\begin{array}{cc}
1, & s_{i}>\varepsilon_{i} \\
\frac{1}{\varepsilon_{i}} s_{i} & -\varepsilon_{i} \leq s_{i} \leq \varepsilon_{i} \\
-1 & s_{i}<-\varepsilon_{i},
\end{array}\right.
$$

where $\varepsilon$ is the thickness of boundary switching layer, $-\varepsilon_{i}$ and $\varepsilon_{i}$ form a continuous approximation region for the switching function, and interval $\left[-\varepsilon_{i}, \varepsilon_{i}\right]$ is called a continuous approximation interval or a boundary layer, where a linear variation with respect to sliding mode surface $s$ exits. Hence, the saturation function $s a t(s / \varepsilon)$ can provide a very smooth control action and reduce the chattering phenomenon of the parallel manipulator.

So the sliding mode control law can be finally expressed as

$$
\mathbf{u}_{1}=\mathbf{M}\left(\ddot{\mathbf{X}}_{d}+\lambda \dot{\mathbf{e}}\right)+\mathbf{C}\left(\dot{\mathbf{X}}_{d}+\lambda \mathbf{e}\right)+\mathbf{G}\left(\mathbf{X}_{d}\right)+\mathbf{K s}+\Lambda s a t\left(\frac{\mathbf{s}}{\varepsilon}\right) .
$$

Using the control law Equation (25), the parallel manipulator can realize the trajectory tracking control with parameters uncertainties and external disturbances. But one of the main drawbacks is that the constant gain $\Lambda$ has to be chosen very large, and the control effort provided by the actuators must also be very large, which may cause chattering to some extent. On account of this, the SMC needs to be improved for good trajectory tracking performance.

3.2. Fuzzy Sliding Mode Control. In order to control the position and orientations of the parallel manipulator effectively, the Fuzzy sliding model control is proposed on the basis of the sliding model control mentioned above. The sliding mode surface function $s$ and the change rate $\dot{\boldsymbol{s}}$ of sliding surface are treated as the input variables, and $u_{f}$ is the output variable.

Referring to the sliding mode control with sliding mode surface s stated in Equation (25), the fuzzy sliding mode control law can be written as follows

$$
u_{2}=\mathbf{M}\left(\ddot{\mathbf{X}}_{d}+\lambda \dot{\mathbf{e}}\right)+\mathbf{C}\left(\dot{\mathbf{X}}_{d}+\lambda \mathbf{e}\right)+\mathbf{G}\left(\mathbf{X}_{d}\right)+\mathbf{K s}+u_{f},
$$

where $u_{f}=\mathbf{N} \operatorname{sat}\left(s_{i} / \varepsilon_{i}\right), \mathbf{N}=\operatorname{diag}\left(n_{1}, n_{2}, n_{3}\right)$, and $n_{1}, n_{2}, n_{3}$ are adjustable constants.

In fuzzy sliding mode control, the switching gain matrix $\mathbf{N}$ is used to compensate the uncertainties that easily arises the chattering and brings the strong oscillation. So in order to decrease the dash, $N$ should be varied with the time according to the tracking errors through the fuzzy logic system. The gains parameters can self-tune based on the sliding surface and its differentiating, which is a big difference from the traditional SMC control method.

The fuzzy tuning technique can modify the output variable gain for a continuous adjustment to reduce the errors without destroying the sliding mode control characteristics. The gain is no longer a fixed constant, but the adjustable constant. So according to the Lyapunov stability theory, the closed-loop system is still asymptotically stable and the trajectory tracking errors can converge to the sliding surface. The sliding switching condition can guarantee the desired trajectory to reach the sliding surface within finite time. Once stabilized, the trajectory will remain on the surface all the time, and thus the upper platform can follow the desired trajectory $[39,40]$. The fuzzy 


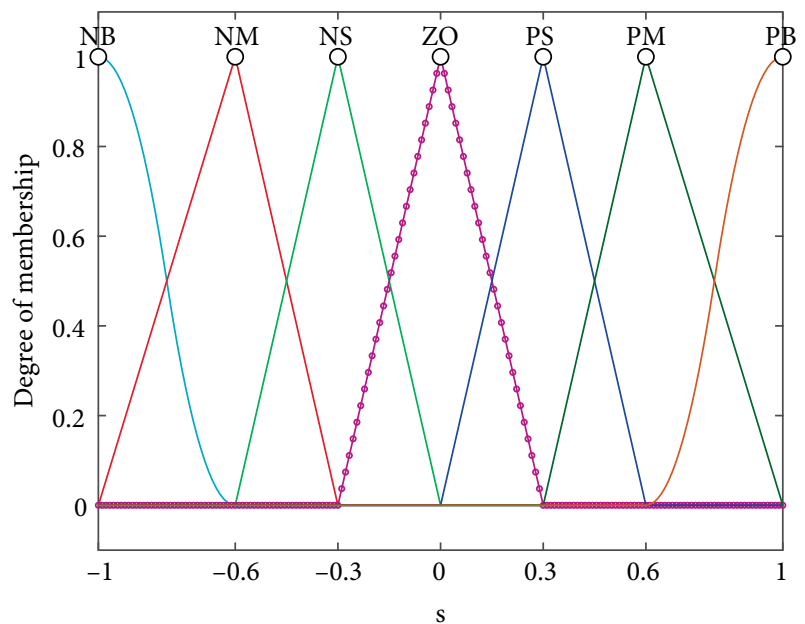

Figure 3: The input membership function of $\mathbf{s}$.

controller mainly consists of three steps: fuzzification, rules evaluation, and defuzzification. In the following, we will introduce in details.

3.2.1. Fuzzification. In the fuzzy system, the sliding surface $\mathbf{s}$ and its derivative $\dot{\mathbf{s}}$ are taken as the input variables of the fuzzy system, and $u_{f}$ is chosen as the output variable of the fuzzy system. Fuzzy sets of input and output of the system are defined with the following symbols, i.e.,

$$
\begin{aligned}
\mathbf{s} & =\left[\begin{array}{lllllll}
\mathrm{NB} & \mathrm{NM} & \mathrm{NS} & \mathrm{ZO} & \mathrm{PS} & \mathrm{PM} & \mathrm{PB}
\end{array}\right] \\
\dot{\boldsymbol{s}} & =\left[\begin{array}{lllllll}
\mathrm{NB} & \mathrm{NM} & \mathrm{NS} & \mathrm{ZO} & \mathrm{PS} & \mathrm{PM} & \mathrm{PB}
\end{array}\right], \\
u_{f} & =\left[\begin{array}{lllllll}
\mathrm{NB} & \mathrm{NM} & \mathrm{NS} & \mathrm{ZO} & \text { PS } & \text { PM } & \mathrm{PB}
\end{array}\right],
\end{aligned}
$$

where NB denotes negative big, NM denotes negative middle, and NS denotes negative small, ZO denotes zero, PS denotes positive small, $\mathrm{PM}$ denotes positive middle, and $\mathrm{PB}$ denotes positive big.

The membership function of input for $\mathbf{s}$ and $\dot{\mathbf{s}}$ are depicted in Figures 3 and 4 , respectively. Triangular shape and $S-Z$ membership functions are utilized and the fuzzy interval is limited to -1 to 1 and -10 to 10 for $\dot{s}$. The membership function of output is shown in Figure 5. Triangular shape and $S-Z$ membership functions are employed and the fuzzy interval is treated as - to 1. The surface viewer is also depicted in Figure 6 about the parameters $\left(\mathbf{s}, \dot{\mathbf{s}}\right.$, and $\left.u_{f}\right)$.

Without loss of generality, there is a proportional coefficient (i.e., $k s, k d s, k u$ ) between the fuzzy interval and the actual interval, as depicted in Figure 7. The coefficients are very important to a nonlinear uncertainties system. The coefficients can affect the dynamic control performance, and they are generally derived from experience.

3.2.2. Rule Evaluation. The control rules must be designed such that the actual trajectory of the states always turns toward and does not cross the sliding surface on phase plane to satisfy existence condition. The min-max composition is chosen as a fuzzy Mamdani inference method.

A fuzzy logic system determines the relationship between inputs and output. This database demonstrates the collection

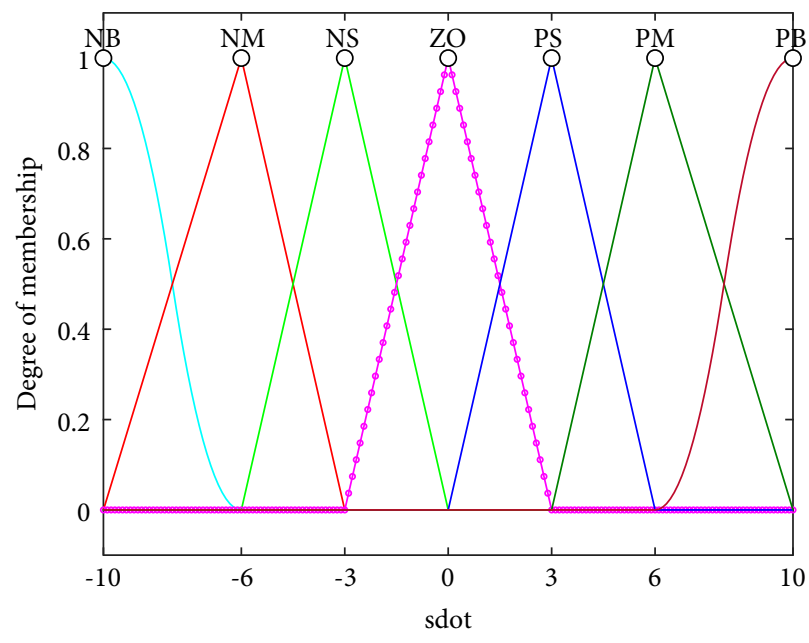

FIGURE 4: The input membership function of $\dot{\mathbf{s}}$.

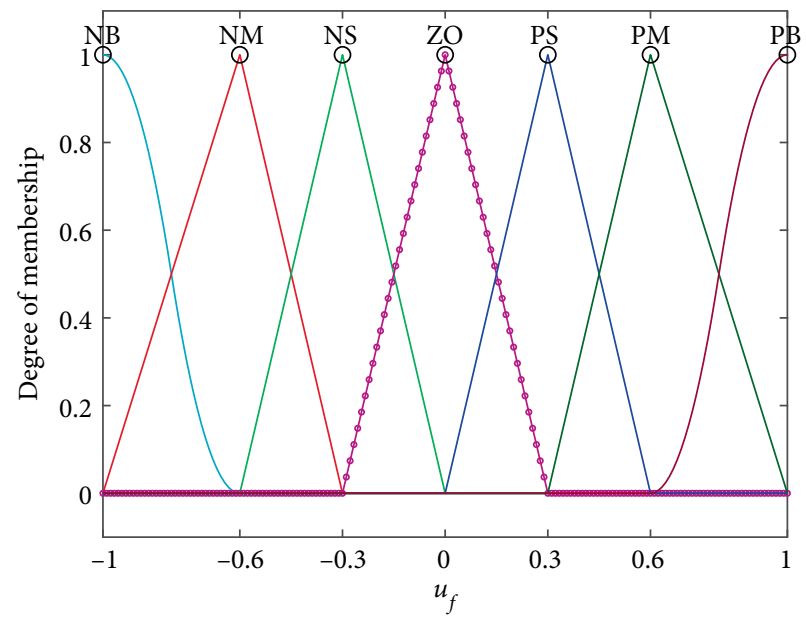

FIGURE 5: The output membership function of $u_{f}$.

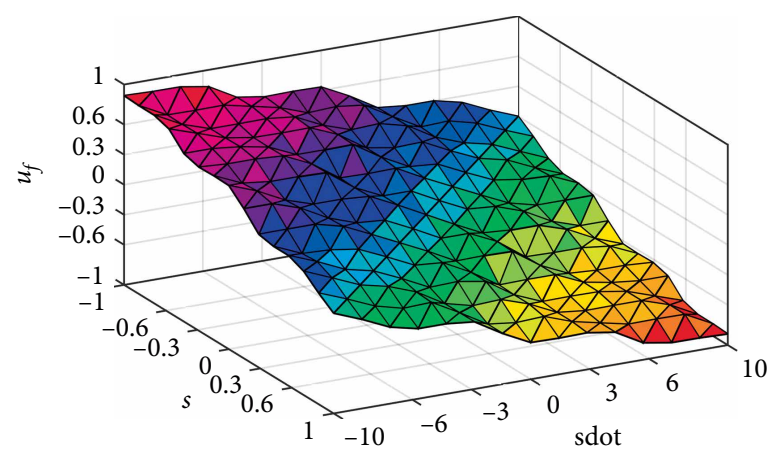

FIGURE 6: The surface viewer of fuzzy variables.

of fuzzy IF-THEN rules. The fuzzy rules can be obtained based on the output acquisition as follows [41]:

Rule 1: IF $\boldsymbol{s}=\mathrm{NB}$, AND $\dot{\boldsymbol{s}}=\mathrm{NB}$, THEN $u_{f}=\mathrm{PB}$

Rule 2: IF $\mathbf{s}=\mathrm{NM}$, AND $\dot{\boldsymbol{s}}=\mathrm{NB}$, THEN $u_{f}=\mathrm{PB}$

Rule 3: IF $\mathbf{s}=\mathrm{NS}$, AND $\dot{\boldsymbol{s}}=\mathrm{NB}, \mathrm{THEN} u_{f}=\mathrm{PM}$

Rule 4: IF $\mathbf{s}=\mathrm{ZO}$, AND $\dot{\boldsymbol{s}}=\mathrm{NB}$, THEN $u_{f}=\mathrm{PM}$ 


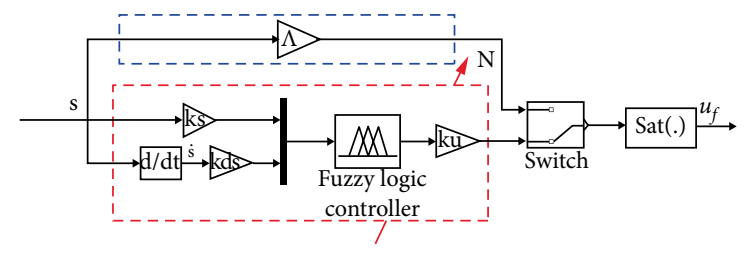

FIgURE 7: The fuzzy control subsystem.

TABLE 1: Fuzzy rule table.

\begin{tabular}{lccccccc}
\hline$\dot{\mathbf{s}}$ & & & \multicolumn{5}{c}{} \\
& NB & NM & NS & ZO & PS & PM & PB \\
\hline NB & PB & PB & PM & PM & PS & PS & ZO \\
NM & PB & PM & PM & PS & PS & ZO & NS \\
NS & PM & PM & PS & PS & ZO & NS & NS \\
ZO & PM & PS & PS & ZO & NS & NS & NM \\
PS & PS & PS & ZO & NS & NS & NM & NM \\
PM & PS & ZO & NS & NS & NM & NM & NB \\
PB & ZO & NS & NS & NM & NM & NB & NB \\
\hline
\end{tabular}

Rule 5: IF $\mathbf{s}=$ PS, AND $\dot{\mathbf{s}}=\mathrm{NB}, \mathrm{THEN} u_{f}=\mathrm{PS}$

Rule 6: IF $\mathbf{s}=\mathrm{PM}$, AND $\dot{\mathbf{s}}=\mathrm{NB}$, THEN $u_{f}=\mathrm{PS}$

Rule 7: IF $\mathbf{s}=\mathrm{PB}$, AND $\dot{\mathbf{s}}=\mathrm{NB}$, THEN $u_{f}=\mathrm{ZO}$

Rule 48: IF $\mathbf{s}=\mathrm{PM}, \mathrm{AND} \dot{\mathbf{s}}=\mathrm{PB}, \mathrm{THEN} u_{f}=\mathrm{NB}$

Rule 49: IF $\mathbf{s}=\mathrm{PB}$, AND $\dot{\mathbf{s}}=\mathrm{PB}, \mathrm{THEN} u_{f}=\mathrm{NB}$

There are 49 fuzzy rules in total, which can be automatically adjusted to achieve satisfactory system response and described in more detail with Table 1.

3.2.3. Defuzzification. The output of the fuzzy controller is fuzzy sets, so it is necessary to convert these fuzzy sets to numerical values. Here, center of gravity (COG) algorithm is used to perform the defuzzification process. According to the membership function, above mentioned rules can be utilized to complete the individual parameter configuration of the input variables $(\mathbf{s}, \dot{\mathbf{s}})$ and output variable $u_{f}$ in their respective domains. The fuzzy control technique can automatically adjust the output variable value $u_{f}$ online against the uncertainties parameters and external disturbances.

3.3. Adaptive Fuzzy Sliding Mode Control. The aforementioned two controllers need to determine the maximum boundary of the uncertainties and external disturbances, but in fact we have difficulty in obtaining the extreme values. If the value of gain matrix is too large, the controller cannot be employed due to the chattering phenomenon. It is with consideration of its complexity that we proposed an adaptive control scheme based on the previous aforementioned approaches to implement the trajectory tracking in this paper.

Adaptive control can be employed to compensate for parametric uncertainties, constraint uncertainties, and bounded external disturbances. That is to say that adaptive controller is insensitive to uncertainties and permits to decrease the requirement for the bound of these uncertainties, and the controller can hold reliability and appropriate stability in the presence of uncertainties and disturbances, which is the main advantage in actual implementation of this method.
The dynamic Equation (6) can be linearized and rewritten in a matrix form as

$$
\begin{aligned}
\mathbf{J}^{T} \boldsymbol{\tau}_{a}= & \mathbf{J}_{p}^{T}\left[\begin{array}{c}
\mathbf{m}_{a} \mathbf{J}_{v p} \\
{ }^{B} \mathbf{I}_{p} \mathbf{J}_{w p}
\end{array}\right] \ddot{\mathbf{X}}+\sum_{i=1}^{4} \mathbf{J}_{v \omega 1 i}^{T}\left[\begin{array}{c}
m_{1 i} \mathbf{J}_{v 1 i} \\
{ }^{B} \mathbf{I}_{1 i} \mathbf{J}_{w i}
\end{array}\right] \ddot{\mathbf{X}} \\
& +\sum_{i=1}^{4} \mathbf{J}_{v \omega 2 i}^{T}\left[\begin{array}{l}
m_{2 i} \mathbf{J}_{v 2 i} \\
{ }^{B} \mathbf{I}_{2 i} \mathbf{J}_{w i}
\end{array}\right] \ddot{\mathbf{X}} \\
& +\mathbf{J}_{p}^{T}\left[{ }^{B} \mathbf{I}_{p} \dot{\mathbf{J}}_{w p}+\left[\left(\mathbf{J}_{w p} \dot{\mathbf{X}}\right) \times\right]\left({ }^{B} \mathbf{I}_{p} \mathbf{J}_{w p}\right)\right] \dot{\mathbf{X}} \\
& +\sum_{i=1}^{4} \mathbf{J}_{v \omega 1 i}^{T}\left[{ }^{B} \mathbf{I}_{1 i} \dot{\mathbf{J}}_{w i}+\left[\left(\mathbf{J}_{w i} \dot{\mathbf{X}}\right) \times\right]\left({ }^{B} \mathbf{I}_{1 i} \mathbf{J}_{w i}\right)\right] \dot{\mathbf{X}} \\
& +\sum_{i=1}^{4} \mathbf{J}_{v \omega 2 i}^{T}\left[{ }^{B} \mathbf{I}_{2 i} \dot{\mathbf{J}}_{w i}+\left[\left(\mathbf{J}_{w i} \dot{\mathbf{X}}\right) \times\right]\left({ }^{B} \mathbf{I}_{2 i} \mathbf{J}_{w i}\right)\right] \dot{\mathbf{X}} \\
& -\left(\mathbf{J}_{p}^{T}\left[\begin{array}{c}
m_{a} \mathbf{g} \\
\mathbf{0}
\end{array}\right]+\sum_{i=1}^{4} \mathbf{J}_{v \omega 1}^{T}\left[\begin{array}{c}
m_{1 i} \mathbf{g} \\
\mathbf{0}
\end{array}\right]+\sum_{i=1}^{4} \mathbf{J}_{v \omega 2}^{T}\left[\begin{array}{c}
m_{2 i} \mathbf{g} \\
\mathbf{0}
\end{array}\right]\right)
\end{aligned}
$$

where $\mathbf{J}_{p}$ is the relations matrix from general velocity to independent velocity parameter and $\mathbf{J}_{p}=\left[\mathbf{J}_{v p} \mathbf{J}_{w p}\right]^{T}, \mathbf{J}_{v w 1 i}$ and $\mathbf{J}_{v w 2 i}$ is the Jacobain matrix of the $i$-th limb, separately. $m_{1 \mathrm{i}}$ and $m_{2 i}$ are the mass of the $i$-th lower and upper limb. ${ }^{B} I_{1 i}$ and ${ }^{B} I_{2 i}$ are the inertia moment of the $i$-th limb with respect to the reference coordinate system $B-x y z$.

Then, we introduce some regression matrices for each inertial force term,

$$
\begin{aligned}
\mathbf{J}^{T} \boldsymbol{\tau}_{a}= & Y_{M p} \Theta_{p}+\sum_{i=1}^{4}\left(\mathrm{Y}_{M i} \Theta_{i}\right)+\mathbf{Y}_{C p} \Theta_{p}+\sum_{i=1}^{4}\left(\mathbf{Y}_{C 1, i} \Theta_{i}\right) \\
& +\sum_{i=1}^{4}\left(\mathbf{Y}_{C 2, i} \Theta_{i}\right)+\mathbf{Y}_{G p} \Theta_{p}+\sum_{i=1}^{4}\left(\mathbf{Y}_{G i} \Theta_{i}\right) .
\end{aligned}
$$

And finally, the above regression matrices can be written into one big matrix form as,

$$
\mathbf{J}^{T} \tau_{a}=\mathbf{Y}(\ddot{\mathbf{X}}, \dot{\mathbf{X}}) \Theta
$$

where $\mathbf{Y}$ is a known $(3 \times 36)$ regression matrix (whose symbolical expression of regression matrix is very complicated and the results document in MATLAB is available here) and $\Theta$ is a $(36 \times 1)$ parameter vector as follows

$$
\begin{gathered}
\mathbf{Y}=\left[\begin{array}{llllll}
\mathbf{Y}_{p} & \mathbf{Y}_{1} & \mathbf{Y}_{2} & \cdots & \mathbf{Y}_{7} & \mathbf{Y}_{8}
\end{array}\right] \in 3 \times 36, \\
\Theta=\left[\begin{array}{llll}
\Theta_{p}^{T} \Theta_{1}^{T} \Theta_{2}^{T} & \cdots & \Theta_{7}^{T} \Theta_{8}^{T}
\end{array}\right]^{T} \in 36 \times 1 .
\end{gathered}
$$

The dynamics parameters which in the case of the parallel manipulator are implemented for simplicity only considering four parameters (i.e., the mass, $m_{i}$, and inertia parameters, $\mathbf{I}_{x x, i}, \mathbf{I}_{y y, i}, \mathbf{I}_{z z, i}$, actually the dynamic parameters should contain ten estimated inertial parameters values as the form [XX XY XZ YY YZ ZZ MX MY MZ M] $]^{\mathrm{T}}$ and the coulomb frictions and viscous frictions of the joints, the details are addressed in $[42,43])$ for each of the nine bodies including the upper 
platform and eight upper and lower limbs of the parallel manipulator [44], i.e.,

$$
\begin{gathered}
\Theta_{i}=\left[\begin{array}{llll}
m_{i} & \mathbf{I}_{x x, i} & \mathbf{I}_{y y, i} & \mathbf{I}_{z z, i}
\end{array}\right]^{T}, \\
\mathbf{Y}_{i}=\mathbf{Y}_{M i}+\mathbf{Y}_{C 1, i}+\mathbf{Y}_{C 2, i}+\mathbf{Y}_{G i}, \\
\mathbf{Y}_{p}=\mathbf{Y}_{M p}+\mathbf{Y}_{C p}+\mathbf{Y}_{G p} .
\end{gathered}
$$

Therefore, the dynamic Equation (6) can be written in a linear regression matrix form as

$$
\mathbf{M} \ddot{\mathbf{X}}+\mathbf{C} \dot{\mathbf{X}}+\mathbf{G}=\mathbf{Y}(\dot{X}, \ddot{X}) \Theta .
$$

Similarly, the following dynamic control equation can be written as

$$
\begin{gathered}
\mathbf{M} \ddot{\mathbf{X}}_{r}+\mathbf{C} \dot{\mathbf{X}}_{r}+\mathbf{G}=\mathbf{Y}\left(\dot{\mathbf{X}}_{r}, \ddot{\mathbf{X}}_{r}, \dot{\mathbf{X}}, \ddot{\mathbf{X}}\right) \Theta, \\
\hat{\mathbf{M}} \ddot{\mathbf{X}}_{r}+\hat{\mathbf{C}} \dot{\mathbf{X}}_{r}+\hat{\mathbf{G}}=\mathbf{Y}\left(\dot{\mathbf{X}}_{r}, \ddot{\mathbf{X}}_{r}, \dot{\mathbf{X}}, \ddot{\mathbf{X}}\right) \hat{\Theta},
\end{gathered}
$$

where $\hat{\Theta}$ is the estimate value of the uncertainty dynamics parameter $\Theta$.

In order to obtain the control law and the adaptive law, the following Lyapunov function candidate is defined as

$$
V=\frac{1}{2} \mathbf{s}^{T} \mathbf{M} \mathbf{s}+\frac{1}{2} \tilde{\Theta}^{T} \boldsymbol{\Gamma}^{-1} \tilde{\Theta} .
$$

It can be shown that the derivative of $V$ with respect to time becomes

$$
\begin{aligned}
\dot{V}= & \mathbf{s}^{T}\left[\mathbf{M} \dot{\mathbf{s}}+\frac{1}{2} \dot{\mathbf{M}} \dot{\mathbf{s}}\right]+\tilde{\Theta}^{T} \boldsymbol{\Gamma}^{-1} \dot{\tilde{\Theta}} \\
= & \mathbf{s}^{T}[\mathbf{M}(\ddot{\mathbf{e}}+\lambda \dot{\mathbf{e}})+\mathbf{C}(\dot{\mathbf{e}}+\lambda \mathbf{e})]+\tilde{\Theta}^{T} \boldsymbol{\Gamma}^{-1} \dot{\tilde{\Theta}} \\
= & \mathbf{s}^{T}\left[\mathbf{M}\left(\ddot{\mathbf{X}}_{d}-\ddot{\mathbf{X}}+\lambda \dot{\mathbf{e}}\right)+\mathbf{C}\left(\dot{\mathbf{X}}_{d}-\dot{\mathbf{X}}+\lambda \mathbf{e}\right)\right]+\tilde{\Theta}^{T} \boldsymbol{\Gamma}^{-1} \dot{\tilde{\Theta}} \\
= & \mathbf{s}^{T}\left[\mathbf{M}\left(\ddot{\mathbf{X}}_{d}+\lambda \dot{\mathbf{e}}\right)+\mathbf{C}\left(\dot{\mathbf{X}}_{d}+\lambda \mathbf{e}\right)-(\mathbf{M} \ddot{\mathbf{X}}+\mathbf{C} \dot{\mathbf{X}})\right] \\
& +\tilde{\Theta}^{T} \boldsymbol{\Gamma}^{-1} \dot{\tilde{\Theta}} .
\end{aligned}
$$

Considering the relation in Equations (14) and (15), the reference velocity and acceleration can be defined as

$$
\begin{aligned}
& \dot{\mathbf{X}}_{r}=\dot{\mathbf{X}}_{d}+\lambda \mathbf{e}=\dot{\mathbf{X}}+\mathbf{s}, \\
& \ddot{\mathbf{X}}_{r}=\ddot{\mathbf{X}}_{d}+\lambda \dot{\mathbf{e}}=\ddot{\mathbf{X}}+\dot{\mathbf{s}} .
\end{aligned}
$$

So the derivate of $V$ can be further expressed as

$$
\dot{V}=\mathbf{s}^{T}\left[\mathbf{M} \ddot{\mathbf{X}}_{r}+\mathbf{C} \dot{\mathbf{X}}_{r}-(\mathbf{M} \ddot{\mathbf{X}}+\mathbf{C} \dot{\mathbf{X}})\right]+\tilde{\Theta}^{T} \boldsymbol{\Gamma}^{-1} \dot{\tilde{\Theta}} .
$$

Combining Equations (6), (37), (41) and (42), yields

$$
\begin{aligned}
\mathbf{M} \ddot{\mathbf{X}}_{r} & +\mathbf{C} \dot{\mathbf{X}}_{r}-(\mathbf{M} \ddot{\mathbf{X}}+\mathbf{C} \dot{\mathbf{X}}) \\
& =\mathbf{M} \dot{\mathbf{s}}+\mathbf{C s}=\mathbf{Y}\left(\dot{\mathbf{X}}_{r}, \ddot{\mathbf{X}}_{r}, \dot{\mathbf{X}}, \ddot{\mathbf{X}}\right) \Theta-\mathbf{F}_{\mathbf{X}} .
\end{aligned}
$$

Substituting the above result into Equation (40), leads to

$$
\dot{V}=\mathbf{s}^{T}\left[\mathbf{Y}\left(\dot{\mathbf{X}}_{r}, \ddot{\mathbf{X}}_{r}, \dot{\mathbf{X}}, \ddot{\mathbf{X}}\right) \Theta-\mathbf{F}_{X}\right]+\tilde{\Theta}^{T} \boldsymbol{\Gamma}^{-1} \dot{\tilde{\Theta}} .
$$

The adaptive fuzzy sliding mode control law can be defined as,

$$
u_{3}=\mathbf{Y}\left(\dot{\mathbf{X}}_{r}, \ddot{\mathbf{X}} r, \dot{\mathbf{X}}, \ddot{\mathbf{X}}\right) \hat{\Theta}+\mathbf{K} \mathbf{s}+u_{f} .
$$

So, Equation (45) can be simplified to,

$$
\dot{V}=-\mathbf{s}^{T} \mathbf{K} \mathbf{s}-\mathbf{s}^{T} \mathbf{N} \mathbf{s}+\mathbf{s}^{T} \mathbf{Y}\left(\dot{\mathbf{X}}_{r}, \ddot{\mathbf{X}} r, \dot{\mathbf{X}}, \ddot{\mathbf{X}}\right) \tilde{\Theta}+\tilde{\Theta}^{T} \boldsymbol{\Gamma}^{-1} \dot{\tilde{\Theta}} .
$$

It is worth noting that the following relation can be utilized,

$$
\left[\mathbf{s}^{T} \mathbf{Y}\left(\dot{\mathbf{X}}_{r}, \ddot{\mathbf{X}}_{r}, \dot{\mathbf{X}}, \ddot{\mathbf{X}}\right) \tilde{\Theta}\right]^{T}=\mathbf{s}^{T} \mathbf{Y}\left(\dot{\mathbf{X}}_{r}, \ddot{\mathbf{X}}_{r}, \dot{\mathbf{X}}, \ddot{\mathbf{X}}\right) \tilde{\Theta} .
$$

The result can be further derived as

$\dot{V}=-\mathbf{s}^{T} \mathbf{K} \mathbf{s}-\mathbf{s}^{T} \mathbf{N} \mathbf{s}+\tilde{\Theta}^{T}\left[\mathbf{Y}\left(\dot{\mathbf{X}}_{r}, \ddot{\mathbf{X}} r, \dot{\mathbf{X}}, \ddot{\mathbf{X}}\right)^{T} \mathbf{s}+\boldsymbol{\Gamma}^{-1} \dot{\tilde{\Theta}}\right]$.

Let $\mathbf{Y}\left(\dot{\mathbf{X}}_{r}, \ddot{\mathbf{X}}_{r}, \dot{\mathbf{X}}, \ddot{\mathbf{X}}\right)^{T} \mathbf{s}+\boldsymbol{\Gamma}^{-1} \dot{\tilde{\Theta}}=0$.

Then, one can obtain

$$
\dot{\tilde{\Theta}}=\boldsymbol{\Gamma Y}\left(\dot{\mathbf{X}}_{r}, \ddot{\mathbf{X}}_{r}, \dot{\mathbf{X}}, \ddot{\mathbf{X}}\right)^{T} \mathbf{s} .
$$

The adaptive law of the unknown dynamic parameters, can be estimated with

$$
\dot{\hat{\Theta}}=-\dot{\tilde{\Theta}}=-\Gamma \mathbf{Y}\left(\dot{\mathbf{X}}_{r}, \ddot{\mathbf{X}}_{r}, \dot{\mathbf{X}}, \ddot{\mathbf{X}}\right)^{T} \mathbf{s} .
$$

Equation (49) is ultimately simplified to

$$
\dot{V}=-\mathbf{s}^{T} \mathbf{K} \mathbf{s}-\mathbf{s}^{T} \mathbf{N} \mathbf{s} .
$$

As $\mathbf{K}$ and $\mathbf{N}$ are a positive definite matrix, $\dot{V}(\mathbf{0})=0$, and $\dot{V}(t) \leq 0$ which is proved to be a semi-negative definite matrix. The sliding surface $\boldsymbol{s}$ will asymptotically converge to zero, and $t \rightarrow \infty, \mathbf{s}=\mathbf{0}$. From Equation (15), we can see that $e$ will asymptotically be zero, $\dot{e}$ also converge to zero, and the upper platform can asymptotically track a desired trajectory in the task space, i.e., $\mathbf{X} \rightarrow \mathbf{X}_{d}$, the closed-loop system will be globally stable in finite time.

The block diagram of the proposed adaptive fuzzy sliding mode controller is illustrated in Figure 8.

\section{Simulation and Discussion}

The parallel manipulator in real environment is fabricated as shown in Figure 9. The implementation of the proposed controller requires controller hardware. Therefore, the computer workstation is based on a frequency $2.8 \mathrm{GHz}$ Intel Core i7-7700HQ processor. It should be mentioned that the closedloop system consists of a six-axis motion controller unit, the four prismatic actuators which are driven by four Delta servo motors ECMA-C20604SS with the output power of 0.4 KiloWatts and rated speed of $3000 \mathrm{r} / \mathrm{min}$ and maximum speed $5000 \mathrm{r} / \mathrm{min}$, and four servo drivers ADSA-B2-0421-B where the incremental photoelectric encoder sensors are mounted coaxially with servo motor to provide position and velocity feedback.

To demonstrate the performance of the proposed control scheme, the adaptive fuzzy sliding-mode controller is first implemented on integrated simulation environment based on 


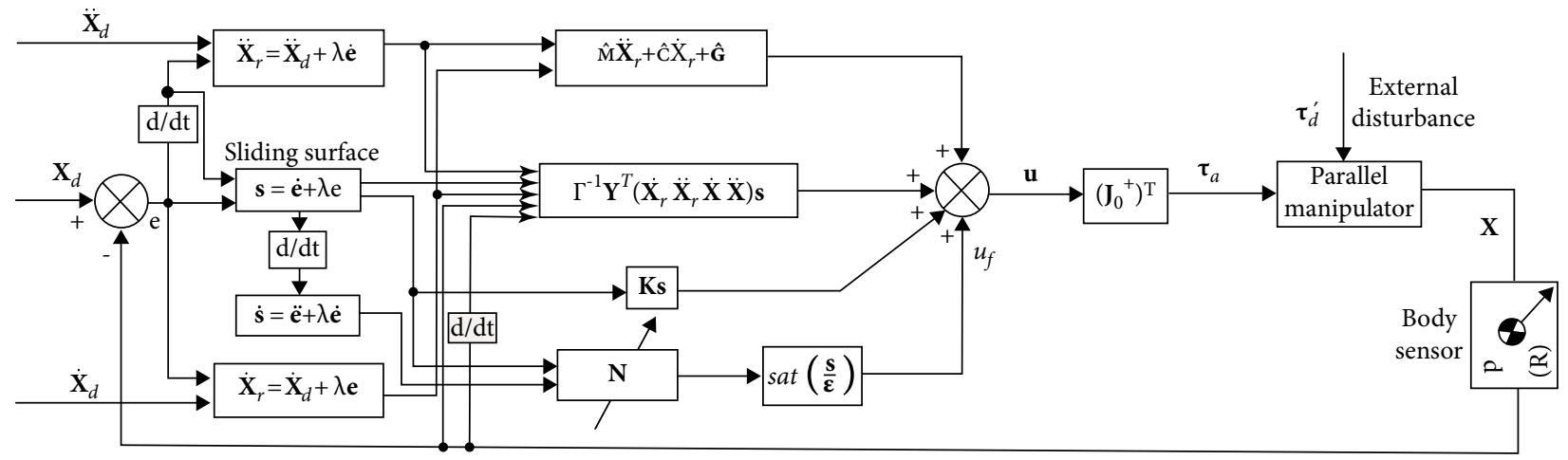

FIGURE 8: Diagram of adaptive fuzzy sliding mode control system.

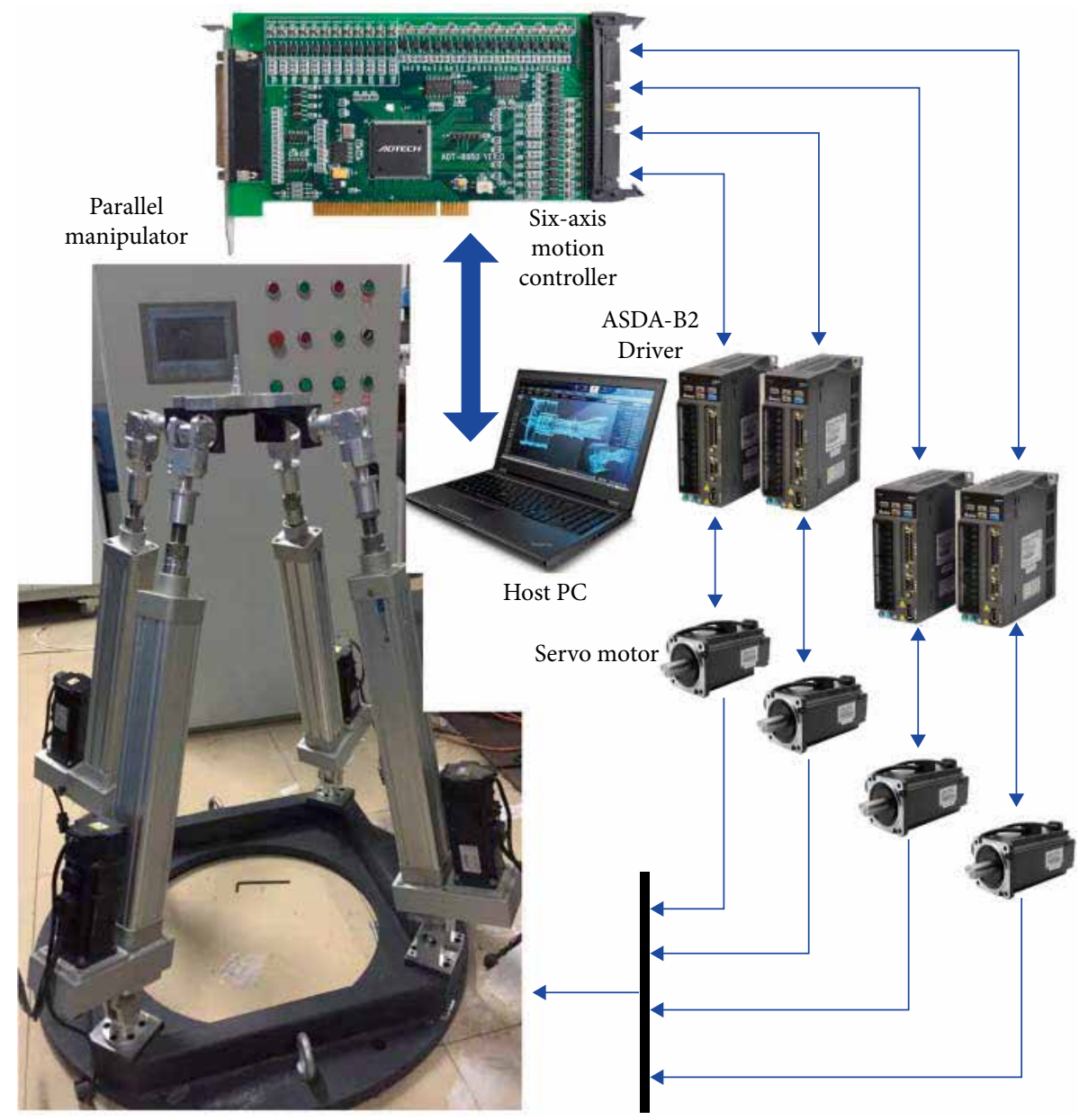

FIGURE 9: The experimental prototype of 3-DOF parallel manipulator.

modular Matlab/Simulink, and real prototype experiments will be conducted in the near future. In addition, for the purpose of comparison, SMC and FSMC control techniques are also implemented. A series of simulation experiments are conducted to illustrate the tracking performance of the adaptive fuzzy sliding mode controller. The geometric parameters used for the parallel mechanism and the location of the gravity center with regard to the world coordinate system $B-x y z$ obtained by the SolidWorks are listed in Table 2 .
Meanwhile, the constant parameters employed in the three controllers are presented in Table 3.

To compare the performance of three controllers in the presence of uncertainties and disturbances, some assuming conditions are considered, i.e., the mass and the moment of inertia in dynamic parameters $\Theta$ are $15 \%$ lower than the real values located in Table 2. Additionally, external disturbances, $\tau_{d}^{\prime}$, applied to the moving platform and they are modeled with the function as follows: 
TABLE 2: Structural parameters of the parallel manipulator.

\begin{tabular}{|c|c|c|}
\hline Variable & Value & Units \\
\hline Radius $r a$ & 0.2 & $\mathrm{~m}$ \\
\hline Radius $r b$ & 0.339 & $\mathrm{~m}$ \\
\hline Upper platform $m_{p}$ & 12.75 & $\mathrm{~kg}$ \\
\hline Upper platform center $e$ & {$\left[\begin{array}{lll}0 & 0 & 0.7\end{array}\right]^{\mathrm{T}}$} & $\mathrm{m}$ \\
\hline Lower limb $m_{1 i}$ & 0.95 & $\mathrm{~kg}$ \\
\hline Lower limb center $e_{11}$ & {$\left[\begin{array}{lll}0 & 0.29 & 0.22\end{array}\right]^{\mathrm{T}}$} & $\mathrm{m}$ \\
\hline Lower limb center $e_{12}$ & {$\left[\begin{array}{lll}-0.29 & 0 & 0.22\end{array}\right]^{\mathrm{T}}$} & $\mathrm{m}$ \\
\hline Lower limb center $e_{13}$ & {$\left[\begin{array}{lll}0 & -0.29 & 0.22\end{array}\right]^{\mathrm{T}}$} & $\mathrm{m}$ \\
\hline Lower limb center $e_{14}$ & {$\left[\begin{array}{lll}0.29 & 0 & 0.22\end{array}\right]^{\mathrm{T}}$} & $\mathrm{m}$ \\
\hline Upper limb $m_{2 \mathrm{i}}$ & 1.1 & $\mathrm{~kg}$ \\
\hline Upper limb center $e_{21}$ & {$\left[\begin{array}{llll}0 & 0.24 & 0.49\end{array}\right]^{\mathrm{T}}$} & $\mathrm{m}$ \\
\hline Upper limb center $e_{22}$ & {$\left[\begin{array}{llll}-0.24 & 0 & 0.49\end{array}\right]^{\mathrm{T}}$} & $\mathrm{m}$ \\
\hline Upper limb center $e_{23}$ & {$\left[\begin{array}{lll}0-0.24 & 0.49\end{array}\right]^{\mathrm{T}}$} & $\mathrm{m}$ \\
\hline Upper limb center $e_{24}$ & {$\left[\begin{array}{lll}-0.24 & 0 & 0.49\end{array}\right]^{\mathrm{T}}$} & $\mathrm{m}$ \\
\hline Upper platform $\mathbf{I}_{\mathrm{xx}}$ & $0.77 \times 10^{-1}$ & $\mathrm{Kg} * \mathrm{~m}^{2}$ \\
\hline Upper platform $\mathbf{I}_{\mathrm{yy}}$ & $1.07 \times 10^{-1}$ & $\mathrm{Kg} * \mathrm{~m}^{2}$ \\
\hline Upper platform $\mathbf{I}_{\mathrm{zz}}$ & $1.83 \times 10^{-1}$ & $\mathrm{Kg} * \mathrm{~m}^{2}$ \\
\hline Lower limb $\mathbf{I}_{\mathrm{xx}, 1 \mathrm{i}}$ & $2.88 \times 10^{-2}$ & $\mathrm{Kg} * \mathrm{~m}^{2}$ \\
\hline Lower limb $\mathbf{I}_{\mathrm{yy}, 1 \mathrm{i}}$ & $2.89 \times 10^{-2}$ & $\mathrm{Kg} * \mathrm{~m}^{2}$ \\
\hline Lower $\operatorname{limb}_{\mathrm{zz}, 1 \mathrm{i}}$ & $0.05 \times 10^{-2}$ & $\mathrm{Kg} * \mathrm{~m}^{2}$ \\
\hline Upper limb $\mathbf{I}_{\mathbf{x x}, 2 \mathrm{i}}$ & $2.65 \times 10^{-2}$ & $\mathrm{Kg} * \mathrm{~m}^{2}$ \\
\hline Upper limb $\mathbf{I}_{\mathrm{yy}, 2 \mathrm{i}}$ & $2.67 \times 10^{-2}$ & $\mathrm{Kg} * \mathrm{~m}^{2}$ \\
\hline Upper limb $\mathbf{I}_{z z, 2 \mathrm{i}}$ & $0.03 \times 10^{-2}$ & $\mathrm{Kg} * \mathrm{~m}^{2}$ \\
\hline Acceleration $\mathbf{g}$ & {$\left[\begin{array}{lll}0 & 0 & -9.807\end{array}\right]^{\mathrm{T}}$} & $\mathrm{m} / \mathrm{s}^{2}$ \\
\hline
\end{tabular}

TABLE 3: The control parameters of three types of controllers.

\begin{tabular}{lc}
\hline Variable & Value \\
\hline $\boldsymbol{\lambda}$ & $\operatorname{diag}[50,20,20]$ \\
$\mathbf{K}$ & $\operatorname{diag}[1000,200,200]$ \\
$\boldsymbol{\Lambda}$ & $\operatorname{diag}[150,40,40]$ \\
$\mathbf{K}_{s}$ & $\operatorname{diag}[10,10,10]$ \\
$\mathbf{K}_{d s}$ & $\operatorname{diag}[0.5,0.5,0.5]$ \\
$\mathbf{K}_{u}$ & $\operatorname{diag}[50,30,30]$ \\
$\boldsymbol{\varepsilon}$ & 0.01 \\
\hline
\end{tabular}

$$
\tau_{d}^{\prime}=2 \times \operatorname{random}(-1,1) .
$$

Specifically, the desired trajectory functions of the upper platform in the workspace are defined to achieve zero velocity and acceleration initially and are as follows

$$
\begin{aligned}
& \alpha(t)=\frac{\pi}{6} \sin (2 t) ; \\
& \beta(t)=\frac{\pi}{6} \cos (2 t) ; \\
& z(t)=0.7+0.02 \sin (2 t) .
\end{aligned}
$$

Furthermore, to quantify the trajectory tracking performance of three controllers, the root square mean error (RSME) is introduced as evaluation index, where the translational displacements and rotational angles are described in separated equation as follows

$$
\operatorname{RSME}_{\alpha}=\sqrt{\frac{1}{m} \sum_{j=1}^{m}\left(X_{z}^{d}(j)-X_{z}(j)\right)^{2}},
$$

$$
\begin{aligned}
& \operatorname{RSME}_{\alpha}=\sqrt{\frac{1}{m} \sum_{j=1}^{m}\left(X_{\alpha}^{d}(j)-X_{\alpha}(j)\right)^{2}}, \\
& \operatorname{RSME}_{\beta}=\sqrt{\frac{1}{m} \sum_{j=1}^{m}\left(X_{\beta}^{d}(j)-X_{\beta}(j)\right)^{2}},
\end{aligned}
$$

where $j$ represents the $j$-th point of tracking trajectory, and $m$ is the total number of data point.

The tracking error of the $i$-th limb is similar to Equation (55), and the obtained results are summarized in Table 4.

As observed in Table 4, the different parameters cases are presented. In comparison with MSC and FMSC, the results illustrate that the FSMC controller has much better root square mean error performance than the SMC controller regardless of which parameter listed above. However, the proposed adaptive controller possesses the best performance compared with other two controllers. Obviously, the displacement parameters (i.e., $z, q_{i}$ ) can follow the desired trajectory in a better performance, and the rotational angle $\alpha$ also has good performance compared with the rotational angle $\beta$ who still need to be improved to some extent.

The trajectory tracking performance of three controllers with the parameters uncertainties and external disturbances are shown in Figures 10-12.

In comparison with Figures 10 and 11, the trajectory tracking errors applied the adaptive fuzzy sliding model controller are a little smaller than the errors associated with sliding mode controller and fuzzy sliding mode controller. In other words, the trajectory tracking performance employed adaptive fuzzy sliding mode controller is better than the sliding mode controller and fuzzy sliding mode controller. As shown in Figure 12, there is a big difference in the response speed and time among three controllers, which indicates the adaptive fuzzy sliding mode controller has better transient response characteristic than other two controllers. It is also concluded that the tracking performance is affected with different controllers, and the proposed adaptive fuzzy sliding mode controller possesses superior trajectory tracking performance to others.

Analogously, the tracking performances of the four actuated limbs with three types of controllers are also depicted in Figures 13-16.

As can be seen from the above figures, the proposed adaptive sliding mode controller combining the sliding mode and fuzzy logic can perform better tracking performance than the two other controllers. For all the trajectories of the actuated limbs, the adaptive fuzzy sliding mode controller shows a good tracking performance. With consideration of uncertainties and disturbances, the adaptive fuzzy sliding mode controller is more robust and insensitive to irregular parametric variation compared with the two other controllers. It is obvious of the second limb and the fourth limb in Figures 14 and 16, the proposed adaptive fuzzy sliding mode controller has a much faster response speed and follows the desired trajectory much better as well.

For verifying the superiority of the proposed adaptive fuzzy sliding mode controller, the tracking error curves of the 
TABLE 4: The comparisons of root square mean error with different controllers.

\begin{tabular}{lccccccc}
\hline \multirow{2}{*}{ Controller } & $z(m m)$ & $\alpha\left(^{\circ}\right)$ & $\beta\left(^{\circ}\right)$ & $q_{1}(m)$ & $q_{2}(m)$ & $q_{3}(m)$ & $q_{4}(m)$ \\
\hline MSC & 0.545968 & 0.918397 & 6.12032 & 0.01207480 & 0.0266254 & 0.00977163 & 0.0173405 \\
FMSC & 0.218587 & 0.825874 & 4.73001 & 0.00969521 & 0.0193492 & 0.00668029 & 0.0140356 \\
AFSMC & 0.377072 & 0.184167 & 2.66612 & 0.00153664 & 0.0094806 & 0.00061928 & 0.0075560 \\
\hline
\end{tabular}

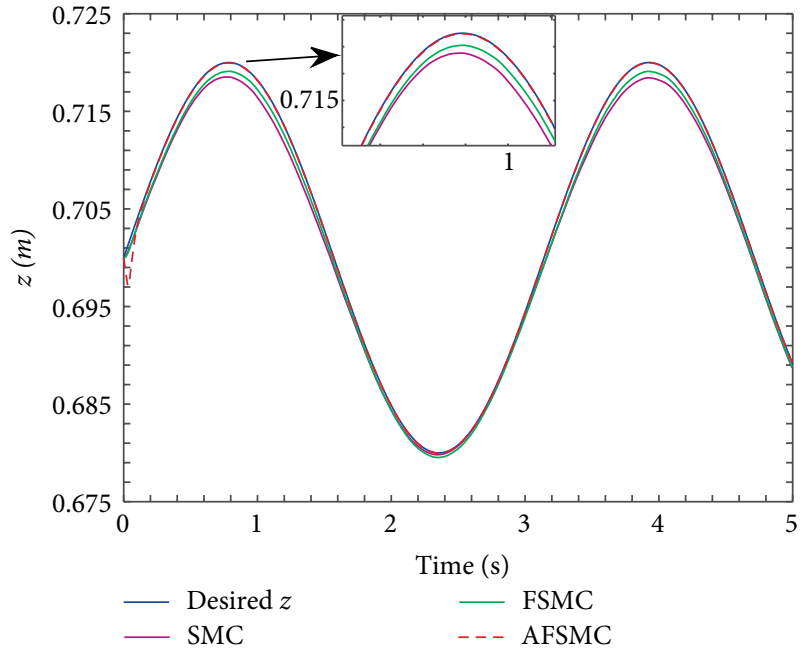

FIGURE 10: Trajectory tracking in $z$ direction of three controllers.

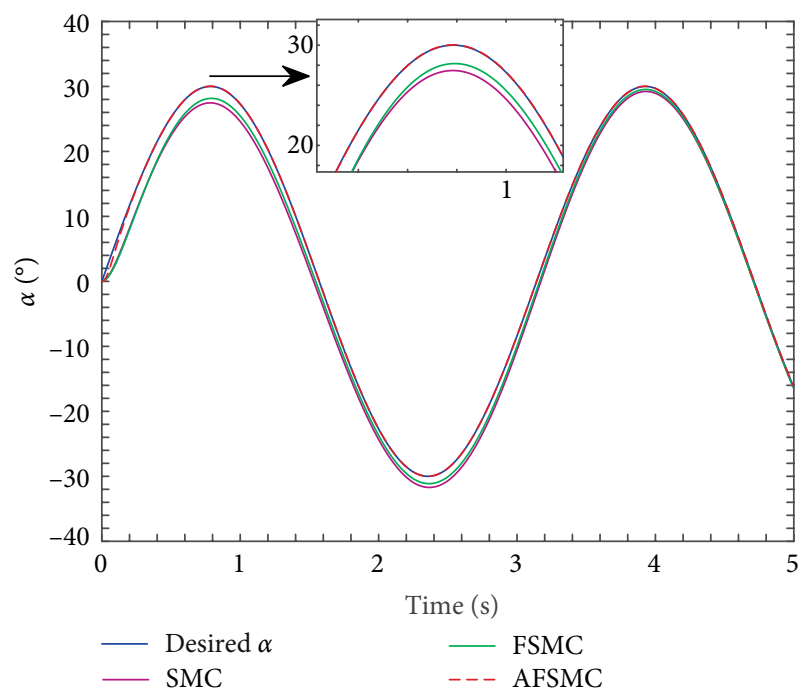

FIgURE 11: Trajectory tracking about rotation angle $\alpha$ of three controllers.

parameters $\left(\begin{array}{lll}z & \alpha & \beta\end{array}\right)$ are drawn, respectively, in Figures 17-19. Through comparative analysis, the tracking error curves based on the sliding mode controller and fuzzy sliding mode controller can be close to zero asymptotically, especially the rotation angle $\beta$. However, the tracking error curves applied the proposed adaptive fuzzy sliding mode controller are asymptotically stable and eventually converge to zero, indicating the closed-loop system is globally stable within finite time. From the trends of the points in simulation process, the

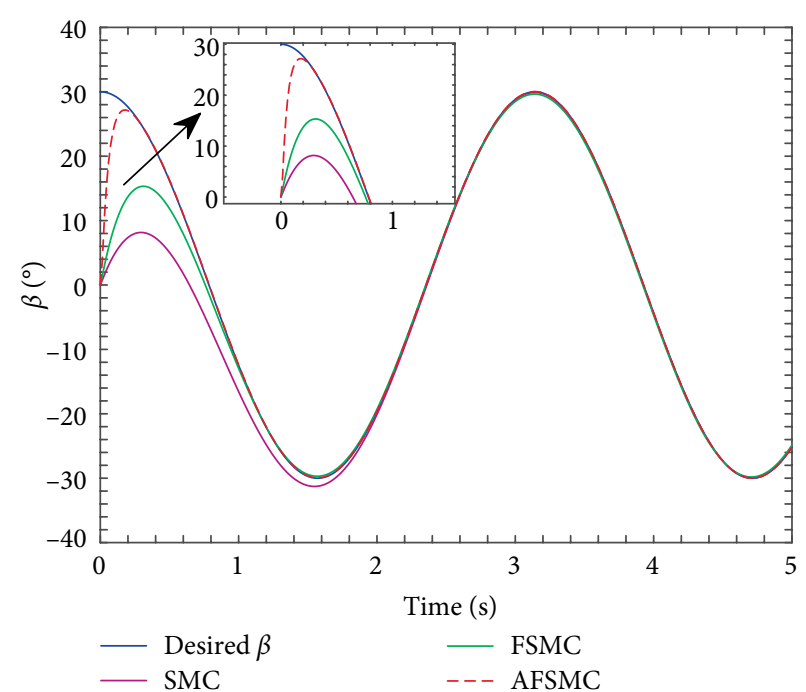

FIGURE 12: Trajectory tracking about rotation angle $\beta$ of three controllers.

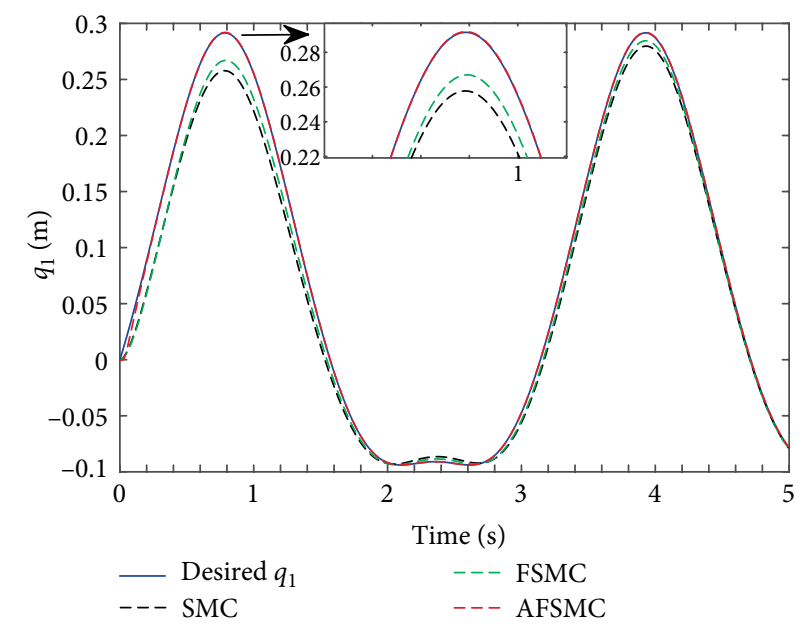

Figure 13: The tracking response of the first limb $q_{1}$.

trajectory tracking can be stable in $0.4 \mathrm{~s}$, which illustrates the adaptive fuzzy sliding mode controller designed in this paper can quickly and accurately estimate the actual value of the parameters to trace the desired trajectory. In particular, the proposed controller can bring about the smallest tracking errors, i.e., approximately converging to zero, compared with sliding mode controller and fuzzy sliding mode controller.

In addition, the trajectory tracking errors under three controllers can be also drawn with box plot, which can display the full range of variation from minimum to maximum, and 


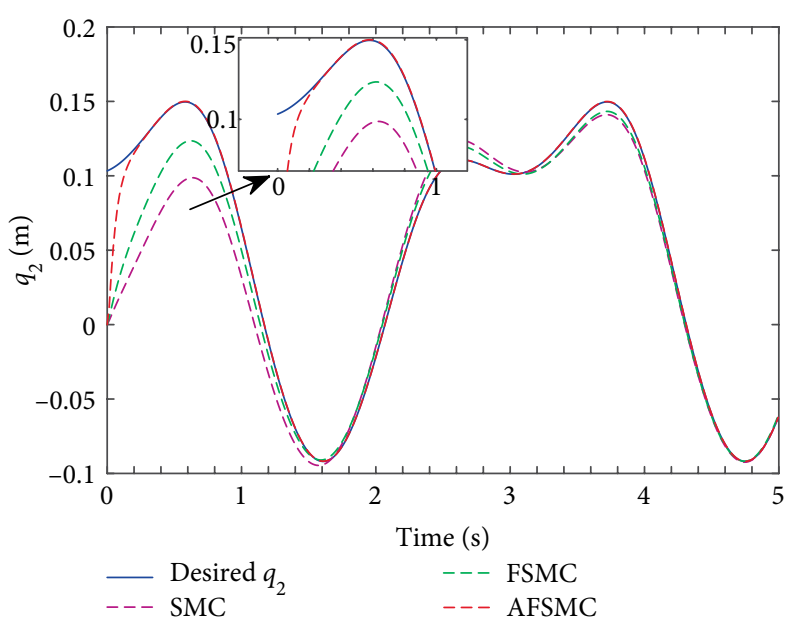

FIGURE 14: The tracking response of the second limb $q_{2}$.

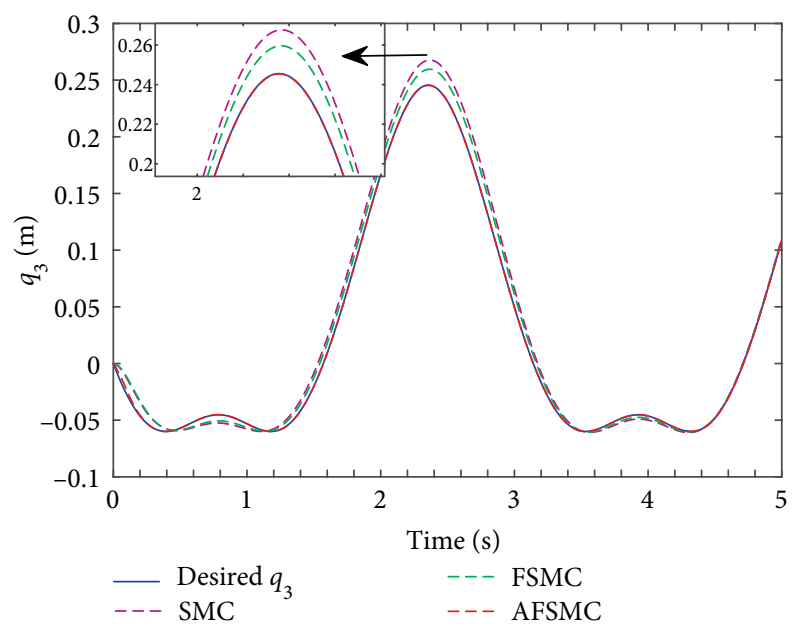

FIGURE 15: The tracking response of the third limb $q_{3}$.

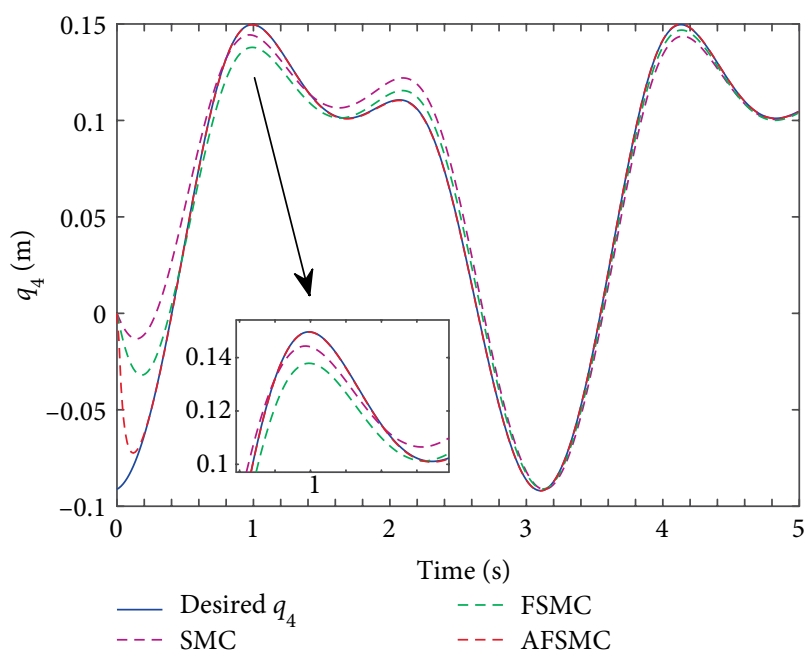

FIGURE 16: The tracking response of the fourth limb $q_{4}$.

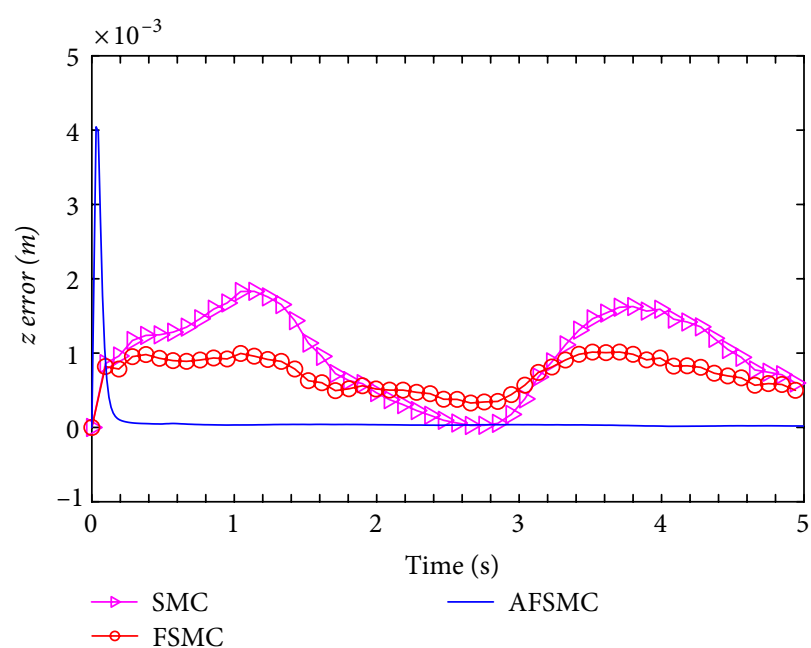

FIGURE 17: The tracking error with desired trajectory in $z$ direction.

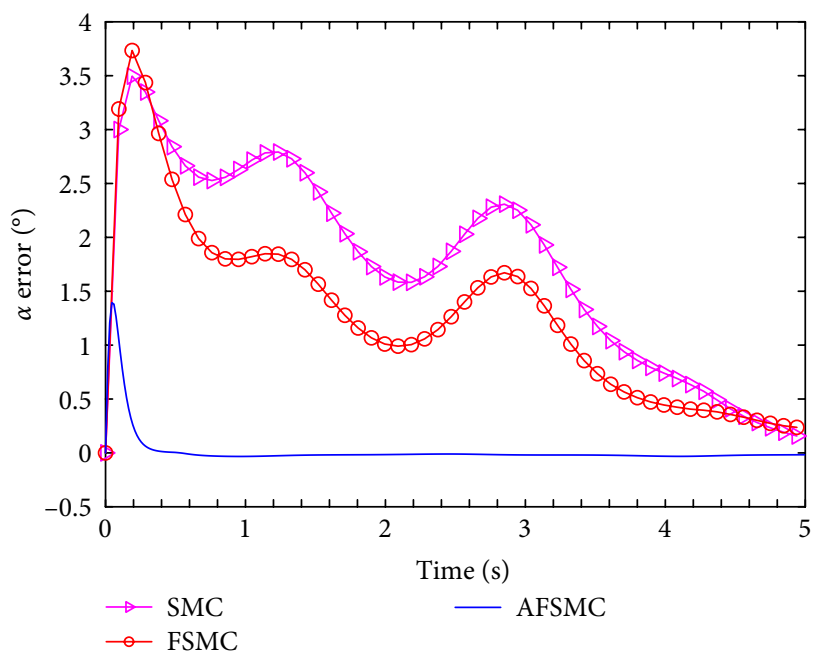

FIGURE 18: The tracking error with desired trajectory with rotation angle $\alpha$.

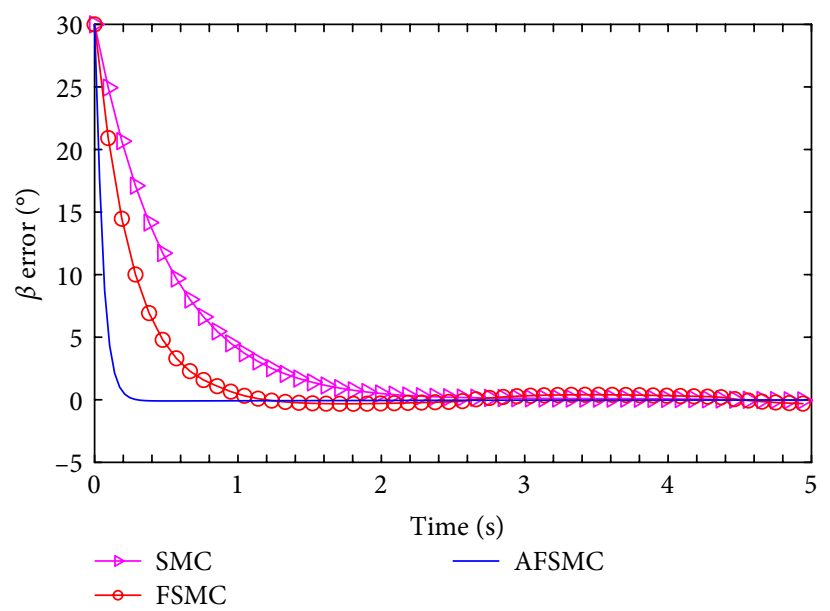

FIGURE 19: The tracking error with desired trajectory with rotation angle $\beta$. 


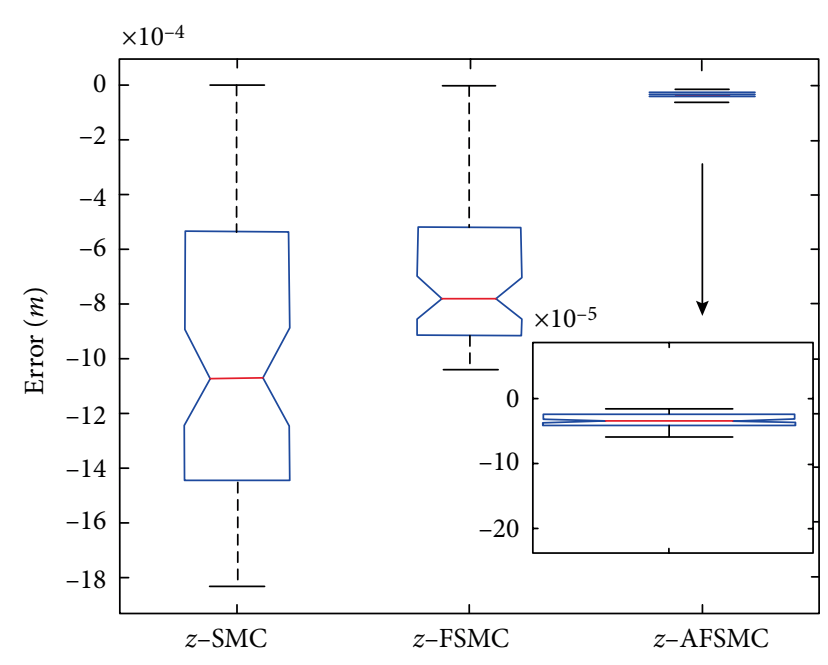

FIGURE 20: The boxplot of parameter $z$ with three controllers.

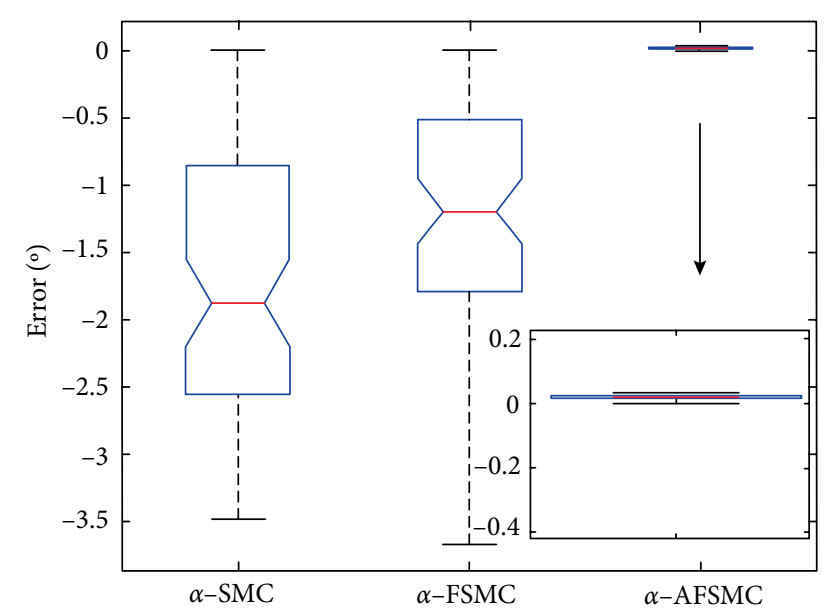

FIGURE 21: The boxplot of parameter $\alpha$ with three controllers.

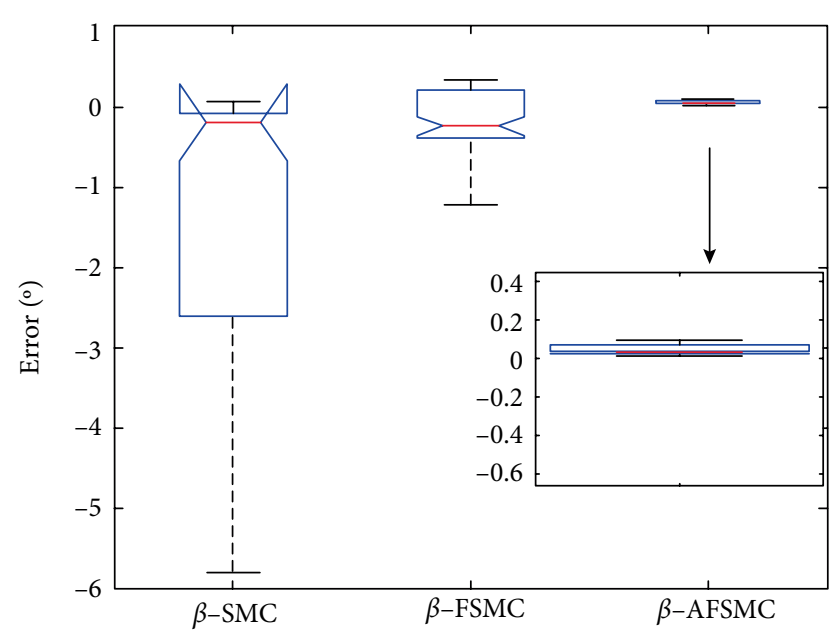

FIgURE 22: The boxplot of parameter $\beta$ with three controllers.

a typical median value. From Figure 20, the datasets $z$ are approximately balanced around zero; evidently the median value in the three cases is near to zero. However, there is

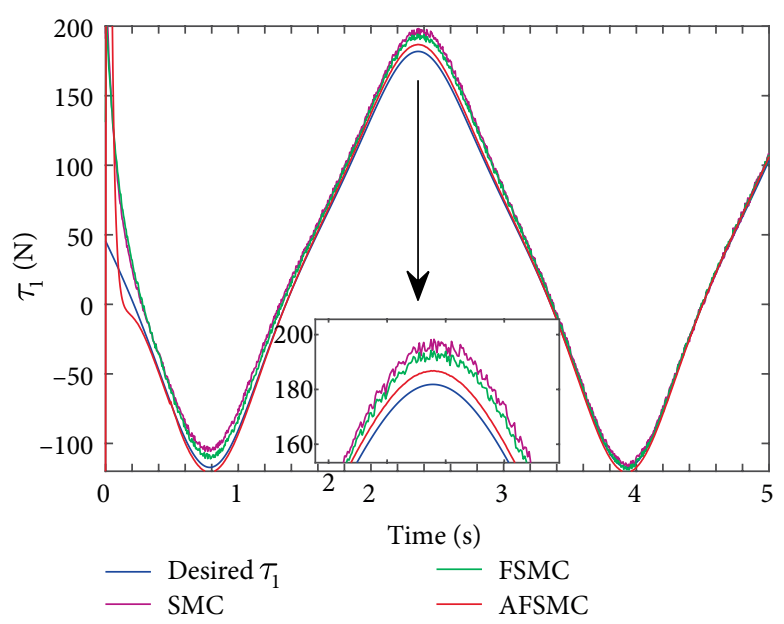

FIGURE 23: The tracking response of the first driving force $\tau_{1}$.

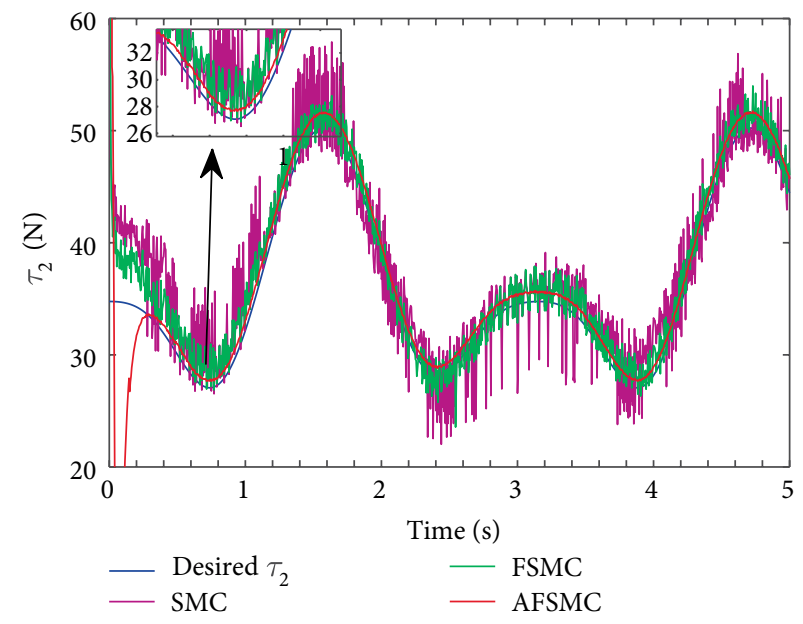

FIGURE 24: The tracking response of the second driving force $\tau_{2}$.

substantially more variation in the SMC controller which ranges approximately from -0.0018 to 0 whereas the one in FSMC controller ranges approximately from -0.001 to 0 . The other Figures 21 and 22 are similar to Figure 20, and we can see that the proposed adaptive controller is superior to the two other controllers in terms of tracking accuracy and stability through comparative analysis, respectively. The trajectory tracking errors of the proposed adaptive fuzzy model controller tend to be zero asymptotically, which illustrate that the proposed controller can effectively compensate the uncertainties caused by parameters variation to deduce the tracking errors.

Moreover, in order to validate the behavior of the mentioned controllers, the input of driving force of the each limb is also very important compared with the desired driving force in Cartesian joint workspace. Figures 23-26 illustrate the tracking errors of four driving force parameters.

As observed in Figures 24-26, the sliding model controller and fuzzy controllers fail to cope with the external disturbances successfully. However, the proposed adaptive fuzzy model controller can eliminate the parameters uncertainties and disturbances, and can be also effectively implemented 


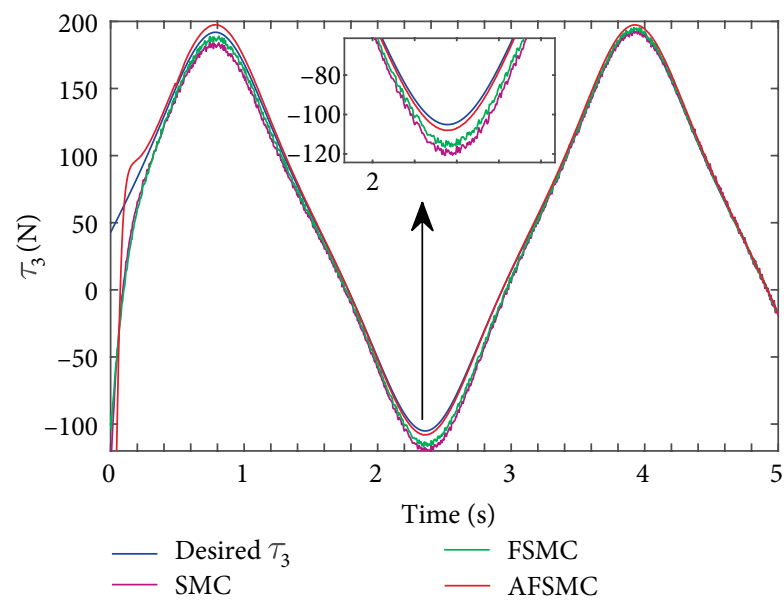

FIGURE 25: The tracking response of the third driving force $\tau_{3}$.

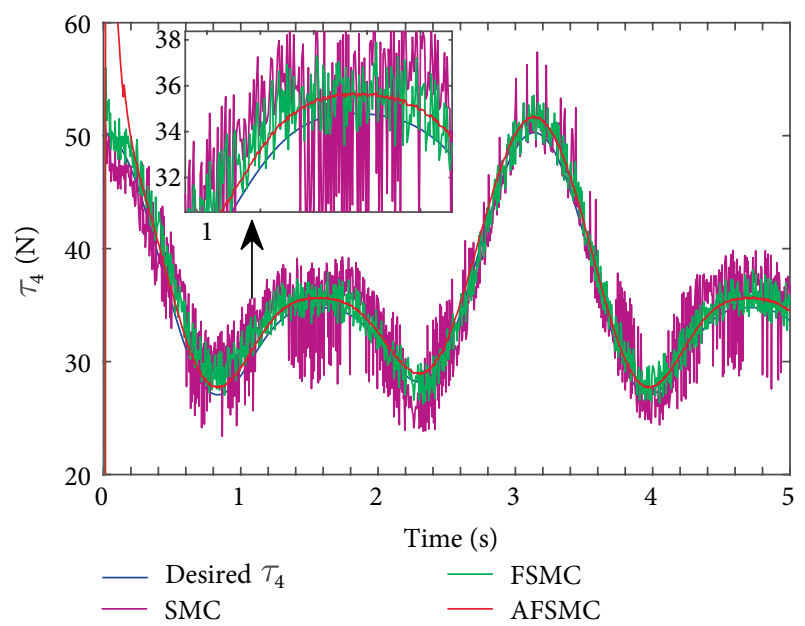

FIGURE 26: The tracking response of the fourth driving force $\tau_{4}$.

online to deal with uncertainties and external disturbances in the closed-loop system. From the enlargements of the localized regions, it can be observed that the chattering or oscillation phenomenon still remains with the sliding mode controller and fuzzy sliding mode controller. If we increase the thickness of boundary layer $\varepsilon$ or decrease the switching gains for reducing the fluctuation of the chattering, then the tracking errors will be increased and the robustness of the closed-loop system will not be guaranteed. Especially from Figures 24 and 26, we can see that the amplitude of the driving force fluctuation of the second and fourth limbs applied the adaptive fuzzy sliding mode controller, which are low in comparison with the other two controllers. Furthermore, the sliding mode controller vibrates more seriously than the fuzzy sliding mode controller which indicates the two controllers presented chattering problem, while adaptive fuzzy sliding mode controller results in a relative smooth driving force. Therefore, the simulation results show that, in comparison with other two controllers, adaptive fuzzy sliding mode controller can deduce the undesirable chattering and improve the robustness of the parallel manipulator regarding parameters uncertainties and irregular external disturbances. In addition, it can be observed that the parameters uncertainties and external disturbances are tremendously compensated for utilizing the proposed adaptive controller. That is to say, the proposed controller can effectively prevent high frequency fluctuation resulting in a more stable trajectory tracking performance, which further demonstrates that the proposed adaptive fuzzy sliding mode controller is superior against the uncertainties and external disturbances compared with the other two controllers mentioned above.

\section{Conclusions}

In this paper, a 3-DOF 2RPU-2SPR parallel manipulator has been presented, which can be applied to high speed machining in serial $X-Y$ linear rail for milling some large heterogeneous complex freedom surfaces in the aerospace field. According to the investigation of this paper, the following contributions can be drawn:

(1) The dynamic model for the 3-DOF parallel manipulator is formulated in the task space utilizing the principle of the virtual work, and the generalized coordinates can be separated into three independent parameters to significantly reduce the closed-loop system complexity in terms of kinematics coupling characteristics.

(2) To achieve high accuracy and stable cooperation, an adaptive fuzzy sliding mode controller for a 3-DOF parallel manipulator is developed in detail. The proposed controller is designed based the sliding mode control and fuzzy logic rules for self-tuning the gains. In the presence of parameters uncertainties and external disturbances with unknown upper bound, a linear regression dynamic equation and adaptive update law are developed for the proposed controller. Besides, the stability of the proposed controller is proven in terms of the Lyapunov theorem.

(3) The simulation experiment results demonstrate that the proposed controller can exhibit accurate and robust trajectory tracking performance without some chattering, which are superior to the results obtained with sliding mode control and fuzzy sliding mode control. Therefore, the proposed adaptive fuzzy sliding mode controller is theoretically effective and practical for the experimental prototype. Further works would be devoted to extending the adaptive controller to unknown parameters identification in practical experiment applications with due consideration of coulomb frictions and viscous frictions term.

\section{Data Availability}

The data used to support the findings of this study are available from the corresponding author upon request. 


\section{Conflicts of Interest}

The authors declared no potential conflicts of interest with respect to the research, authorship, and/or publication of this article.

\section{Acknowledgements}

The authors would like to acknowledge the financial support of the Fundamental Research Funds for the Central Universities under Grants No. 2018JBZ007, No. 2018YJS136 and No. 2017YJS158, China Scholarship Council (CSC) under Grant No. 201807090079, the Natural Sciences and Engineering Research Council of Canada (NSERC), and the York Research Chairs (YRC) program. Meanwhile the author, Haiqiang Zhang, is grateful to advanced robotics and mechatronics laboratory in York University and the science librarian John Dupuis.

\section{Supplementary Materials}

Supplementary 1: The regression matrix of Equation (30) in manuscript. (Supplementary Materials)

\section{References}

[1] M. Luces, J. K. Mills, and B. Benhabaib, "A review of redundant parallel kinematic mechanisms," Journal of Intelligent and Robotic Systems, vol. 86, no. 2, pp. 175-198, 2017.

[2] X. D. Jin, Y. F. Fang, H. B. Qu, and S. Guo, "A class of novel 4-DOF and 5-DOF generalized parallel mechanisms with high performance," Mechanism and Machine Theory, vol. 120, pp. 57-72, 2018.

[3] Z. Gao and D. Zhang, "Performance analysis, mapping, and multiobjective optimization of a hybrid robotic machine tool," IEEE Transactions on Industrial Electronics, vol. 62, no. 1, pp. 423-433, 2015.

[4] H. J. Yap, J. G. Ng, Z. A. Zakaria, Z. Taha, S.-W. Chang, and K. S. Yap, "Design and development of 6-DOF system for virtual bicycle," Movement, Health and Exericise, vol. 5, no. 2, pp. 31-39, 2016.

[5] W. D. Wang, H. J. Song, Z. Y. Yan, and L. N. Sun, "A universal index and an improved PSO algorithm for optimal pose selection in kinematic calibration of a novel surgical robot," Robotics and Computer-Integrated Manufacturing, vol. 50, pp. 90-101, 2018.

[6] J. Borras and A. M. Dollar, "Analyzing dexterous hands using a parallel robots framework," Autonomous Robots, vol. 36, no. 1 , pp. 169-180, 2014

[7] H. L. Wang, "Adaptive control of robot manipulators with uncertain kinematics and dynamics," IEEE Transaction on Automatic Control, vol. 62, no. 2, pp. 948-954, 2017.

[8] J. Wu, X. M. Chen, T. M. Li, and L. P. Wang, "Optimal design of a 2-DOF parallel manipulator with actuation redundancy considering kinematics and natural frequency," Robotics and Computed-Integrated Manufacturing, vol. 29, no. 1, pp. 80-85, 2013.
[9] D. Liang, Y. M. Song, and T. Sun, "Nonlinear dynamic modeling and performance analysis of a redundantly actuated parallel manipulator with multiple actuation modes based on FMD theory," Nonlinear Dynamics, vol. 89, no. 1, pp. 391-428, 2017.

[10] M. Sharifzaden, M. T. Masouleh, A. Kalhor, and P. Shahverdi, "An experimental dynamic identification and control of an overconstrained 3-DOF parallel mechanism in presence of variable friction and feedback delay," Robotics and Autonomous Systems, vol. 102, pp. 27-43, 2018.

[11] K. Guo, Y. P. Pan, and H. Y. Yu, "Composite learning robot control with friction compensation: a neural network-based approach," IEEE Transactions on Industrial Electronics, vol. 66, no. 10, pp. 7841-7851, 2019.

[12] S. Roy, S. B. Roy, and I. N. Kar, "Adaptive-robust control of euler-lagrange systems with linearly parametrizable uncertainty bound," IEEE Transactions on Control Systems Technology, vol. 26, no. 5, pp. 1842-1850, 2018.

[13] Y. X. Su, B. Y. Duan, and C. H. Zheng, "Nonlinear PID control of a six-DOF parallel manipulator," IEE Proceedings - Control Theory and Applications, vol. 151, no. 1, pp. 95-102, 2004.

[14] W. W. Shang and S. Cong, "Nonlinear computed torque control for a high-speed planar parallel manipulator," Mechatronics, vol. 19, no. 6, pp. 987-992, 2009.

[15] D. Zhang and B. Wei, "Convergence performance comparisons of PID, MRAC, and PID + MRAC hybrid controller," Frontiers of Mechanical Engineering, vol. 11, no. 2, pp. 213-217, 2016.

[16] L. Sheng and W. Li, "Optimization design by genetic algorithm controller for trajectory control of a 3-RRR parallel robot," Algorithms, vol. 11, no. 7, pp. 1-13, 2018.

[17] T. S. Zhan and C. C. Kao, "Modified PSO method for robust control of 3RPS parallel manipulators," Mathematical Problems in Engineering, vol. 2010, Article ID 302430, 25 pages, 2010.

[18] I. Buzurovic, "Advanced control methodologies in parallel robotic systems," Advances in Robotics and Automation, vol. 6, pp. 1-2, 2012.

[19] V. T. Nguyen, C. Y. Lin, S. F. Su, and Q. V. Tran, "Adaptive chattering free neural network based sliding mode control for trajectory tracking of redundant parallel manipulators," Asian Journal of Control, vol. 21, no. 2, pp. 908-923, 2019.

[20] J. He, M. Z. Luo, Q. Q. Zhang, and J. H. Zhao, "Adaptive fuzzy sliding mode controller with nonlinear observer for redundant manipulators handling varying external force," Journal of Bionic Engineering, vol. 13, pp. 600-611, 2016.

[21] X. Yang, L. M. Zhu, Y. B. Ni, and H. T. Hai, "Modified robust dynamic control for a diamond parallel robot," IEEE/ASME Transactions on Mechatronics, vol. 24, no. 3, pp. 959-968, 2019.

[22] J. Cazalilla, M. Valles, V. Mata, and M. Diaz-Rodriguez, "Adaptive control of a 3-DOF parallel manipulator considering payload handling and relevant parameter models," Robotics and Computer-Integrated Manufacturing, vol. 30, no. 5, pp. 468-477, 2014.

[23] R. Babaghasabha, M. A. Khosravi, and H. D. Taghirad, "Adaptive control of KNTU planar cable-driven parallel robot with uncertainties in dynamic and kinematic parameters," in Cable-Driven Parallel Robots, A. Pott and T. Bruckmann, Eds., vol. 32 of Mechanisms and Machine Science, pp. 145-159, Springer, Cham, 2015. 
[24] W. W. Shang, S. Cong, and Yuan Ge, "Adaptive computed torque control for a parallel manipulator with redundant actuation," Robotica, vol. 30, pp. 457-466, 2012.

[25] T. D. Le, H. J. Kang, and Y. S. Suh, "Chattering-free neurosliding mode control of 2-DOF planar parallel manipulators," International Journal of Advanced Robotic Systems, vol. 10, pp. 1-15, 2013.

[26] Z. Qi, J. E. Mclnroy, and F. Jasfri, “Trajectory tracking with parallel robots using low chattering, fuzzy sliding mode controller," Journal of Intelligent and Robotic Systems, vol. 48, no. 3, pp. 333-356, 2007.

[27] A. Filabi and M. Yaghoobi, "Fuzzy adaptive sliding mode control of 6 DOF parallel manipulator with electromechanical actuators in cartesian space coordinates," Communications on Advanced Computational Science with Applications, vol. 2015, no. 1, pp. 1-21, 2015.

[28] G. L. Tao, C. Shang, and D. Y. Meng, "Adaptive robust posture control of a 3-RPS pneumatic parallel platform with unknown deadzone," Mathematical Problems in Engineering, vol. 2016, Article ID 2034923, 16 pages, 2016.

[29] H. Navvabi and A. H. D. Markazi, "Position control of stewart manipulator using a new extended adaptive fuzzy sliding mode controller and observer (E-AFSMCO)," Journal of the Franklin Institute, vol. 355, no. 5, pp. 2583-2609, 2018.

[30] H. Q. Zhang, H. R. Fang, D. Zhang, X. L. Luo, and Q. Zou, "Forward kinematics and workspace determination of a novel redundantly actuated parallel manipulator," International Journal of Aerospace Engineering, vol. 2019, Article ID 4769174, 14 pages, 2019.

[31] G. L. Wu, S. P. Bai, J. A. Kepler, and S. Caro, "Error modeling and experimental validation of a planar 3-PPR parallel manipulator with joint clearances," Journal of Mechanisms and Robotics, American Society of Mechanical Engineers, vol. 4, no. 4, pp. 1-8, 2012.

[32] L. W. Lee, H. H. Chiang, and I. H. Li, "Development and control of a pneumatic-actuator 3-DOF translational parallel manipulator with robot vision," Sensors, vol. 19, no. 6, pp. 1-26, 2019.

[33] L. Carbonari, "Simplified approach for dynamics estimation of a minor mobility parallel robot," Mechatronics, vol. 33, pp. 76-84, 2015.

[34] H. Abdellatif and B. Heimann, "Computational efficient inverse dynamics of 6-DOF fully parallel manipulators by using the Lagrangian formalism," Mechanism and Machine Theory, vol. 44, no. 1, pp. 192-207, 2009.

[35] B. Dasgupta and P. Choudhury, "A general strategy based on the newton-euler approach for the dynamic formulation of parallel manipulators," Mechanism and Machine Theory, vol. 34, no. 6, pp. 801-824, 1999.

[36] Y. Yun and Y. Li, "Design and analysis of a novel 6-DOF redundant actuated parallel robot with compliant hinges for high precision positioning," Nonlinear Dynamics, vol. 61, no. 4, pp. 829-845, 2010

[37] H. Q. Zhang, H. R. Fang, B. S. Jiang, and S. G. Wang, "Dynamic performance evaluation of a redundantly actuated and overconstrained parallel manipulator," International Journal of Automation and Computing, vol. 16, no. 3, pp. 274-285, 2019.

[38] Frank L. Lewis, Darren M. Dawson, and Chaouki T. Abdallah, Robot Manipulator Control: Theory and Practice, CRC Press, 2003.
[39] I. Nazari, A. Hosainpour, F. Piltan, S. Emamzadeh, and M. Mirzaie, "Design sliding mode controller with parallel fuzzy inference system compensator to control of robot manipulator," International Journal Intelligent System and Applications, vol. 4, pp. 63-75, 2014.

[40] S. H. Wen, J. H. Chen, G. Q. Qin, and Q. G. Zhu, "An improved fuzzy model predictive control algorithm based on the force/ position control structure of the five-degree of freedom redundant actuation parallel robot," International Journal of Advanced Robotic Systems, vol. 9, pp. 1-8, 2018.

[41] H. Liu, S. G. Li, H. X. Wang, and Y. G. Sun, "Adaptive fuzzy control for a class of unknown fractional-order neural networks subject to input nonlinearities and dead-zones," Information Sciences, vol. 4, pp. 30-45, 2018.

[42] G. El-Ghazaly, M. Gouttefarde, and V. Creuze, Adaptive Terminal Sliding Mode Control of a Redundantly-Actuated CableDriven Parallel Manipulator: CoGiRo, vol. 32, Mechanisms and Machine Science, pp. 179-200, 2014.

[43] W. Khalil and E. Dombre, Modeling, Identification and Control of Robots, Butterworth-Heinemann Book, pp. 251-256, 2004.

[44] E. Yime, R. Saltaren, C. Garcia, and J. M. Sabater, "Robot based on task-space dynamical model," IET Control Theory and Application, vol. 5, no. 18, pp. 2111-2119, 2011. 